TRANSACTIONS OF THE

AMERICAN MATHEMATICAL SOCIETY

Volume 353, Number 6, Pages 2131-2170

S 0002-9947(01)02741-6

Article electronically published on January 3, 2001

\title{
ON SUPPORTS AND ASSOCIATED PRIMES OF MODULES OVER THE ENVELOPING ALGEBRAS OF NILPOTENT LIE ALGEBRAS
}

\author{
BORIS ŠIROLA
}

\begin{abstract}
Let $\mathfrak{n}$ be a nilpotent Lie algebra, over a field of characteristic zero, and $\mathcal{U}$ its universal enveloping algebra. In this paper we study: (1) the prime ideal structure of $\mathcal{U}$ related to finitely generated $\mathcal{U}$-modules $V$, and in particular the set Ass $V$ of associated primes for such $V$ (note that now Ass $V$ is equal to the set Annspec $V$ of annihilator primes for $V$ ); (2) the problem of nontriviality for the modules $V / \mathcal{P} V$ when $\mathcal{P}$ is a (maximal) prime of $\mathcal{U}$, and in particular when $\mathcal{P}$ is the augmentation ideal $\mathcal{U} \mathfrak{n}$ of $\mathcal{U}$. We define the support of $V$, as a natural generalization of the same notion from commutative theory, and show that it is the object of primary interest when dealing with (2). We also introduce and study the reduced localization and the reduced support, which enables to better understand the set Ass $V$. We prove the following generalization of a stability result given by W. Casselman and M. S. Osborne in the case when $\mathfrak{N}, \mathfrak{N}$ as in the theorem, are abelian. We also present some of its interesting consequences.

Theorem. Let $\mathfrak{Q}$ be a finite-dimensional Lie algebra over a field of characteristic zero, and $\mathfrak{N}$ an ideal of $\mathfrak{Q}$; denote by $U(\mathfrak{N})$ the universal enveloping algebra of $\mathfrak{N}$. Let $V$ be a $\mathfrak{Q}$-module which is finitely generated as an $\mathfrak{N}$-module. Then every annihilator prime of $V$, when $V$ is regarded as a $U(\mathfrak{N})$-module, is $\mathfrak{Q}$-stable for the adjoint action of $\mathfrak{Q}$ on $U(\mathfrak{N})$.
\end{abstract}

\section{INTRODUCTION}

Let $G$ be a connected semisimple Lie group with finite center. Let $K$ be a maximal compact subgroup of $G$, and $Q$ a minimal parabolic subgroup of $G$ with the corresponding Langlands decomposition $Q=M A N$. Let $\mathfrak{g}_{0}, \mathfrak{k}_{0}, \mathfrak{q}_{0}, \mathfrak{m}_{0}, \mathfrak{a}_{0}$ and $\mathfrak{n}_{0}$ be the Lie algebras of $G, K, Q, M, A$ and $N$, respectively. By $\mathfrak{g}, \mathfrak{k}$, etc., denote the corresponding complexifications. Also, let $\mathcal{U}$ denote the universal enveloping algebra $U(\mathfrak{n})$ of $\mathfrak{n}$, and $\mathcal{A}$ the augmentation ideal $\mathcal{U} \mathfrak{n}$ of $\mathcal{U}$. Now we can state the following important result.

Theorem (Casselman). Let $V$ be an admissible finitely generated ( $\mathfrak{g}, K)$-module. Then $\mathcal{A} V \neq V$.

Received by the editors February 5, 1999 and, in revised form, June 3, 1999 and January 31, 2000.

2000 Mathematics Subject Classification. Primary 17B35, 16P50.

Key words and phrases. Nilpotent Lie algebra, semisimple Lie algebra, parabolic subalgebra, universal enveloping algebra, prime ideal, augmentation ideal, associated prime, annihilator prime, foundation prime, localization, support, reduced localization, reduced support, (co)adjoint action, stable prime, coadjoint orbit.

The author was supported by Fulbright Grant No. 22676, and in part by the Ministry of Science and Technology, Republic of Croatia. 
As a consequence of this theorem one obtains that if $V$ is moreover irreducible then $V$ embeds in a principal series representation. This is the famous Casselman's subrepresentation theorem. Although the statement of the above theorem is purely algebraic, Casselman's original proof is analytic in nature and deals with the asymptotic expansions of matrix coefficients at infinity (see [CM] ). The first algebraic proof are due to A. Beilinson and J. Bernstein. They obtained the above theorem as a corollary of a more general result proved via $\mathcal{D}$-module theory techniques [BB]. Also, Casselman and M. S. Osborne gave an algebraic proof, but under the strong restriction that the nilpotent radical $\mathfrak{n}$ is abelian [CO].

In [C, p.560] Casselman stated that the known results on associated primes for $\mathcal{U}$-modules are insufficient for extending the techniques from [CO] to the case of nonabelian $\mathfrak{n}$. The main purpose of the present paper is to establish some general prime ideal structure results for finitely generated modules over the enveloping algebras of finite-dimensional nilpotent Lie algebras defined over an algebraically closed field of characteristic zero. Further, we generalize one stability result given by Casselman and Osborne in the commutative setting, to the noncommutative case (see Theorem C), and give a number of interesting consequences of it.

Let $\mathcal{R}$ be any left Noetherian ring with identity and $M$ a finitely generated $\mathcal{R}$-module. If we want to understand the structure of $M$, it is often useful to know something about certain special kinds of prime ideals of $\mathcal{R}$ which are closely related to $M$, such as the annihilator primes Annspec $M$ and the associated primes Ass $M$. In [ัS1] we introduced the set w-Ass $M$ of primes w-associated to $M$ (for $M$ not necessarily finitely generated). Also, in [ك̌2] we introduced the set Fnd $M$ of foundation primes for $M$. Now, all these sets of primes are finite and it turns out that Ass $M \subseteq$ w-Ass $M=$ Annspec $M \subseteq$ Fnd $M$ (see Sect. 1).

Denote by $\mathcal{K}$ the class of Noetherian CSG-rings in which every prime ideal is moreover completely prime. (We say that $\mathcal{R}$ is a CSG-ring, or that $\mathcal{R}$ satisfies the CSG-condition, if every ideal of $\mathcal{R}$ has a centralizing sequence of generators; see Sect. 0, Definition.) Of course, every commutative Noetherian ring is from $\mathcal{K}$. But, also, $\mathcal{K}$ contains another interesting class of rings. Namely, let $\mathfrak{n}$ be any finite-dimensional nilpotent Lie algebra defined over a field $k$ of characteristic zero, $U(\mathfrak{n})$ its universal enveloping algebra and $I$ an arbitrary two-sided ideal of $U(\mathfrak{n})$. Define the quotient algebra $\Lambda:=U(\mathfrak{n}) / I$. Then by the results of J. Dixmier $[\mathrm{D}$, 3.7.2 Thm.], J. C. McConnell [McC] and Y. Nouazé and P. Gabriel [NG] one knows that $\Lambda$ is an AR (Artin-Rees) $k$-algebra from $\mathcal{K}$. Further, by the result of P. F. Smith [S] it follows that for any $\mathcal{R} \in \mathcal{K}$ and a prime $\mathcal{P}$ of $\mathcal{R}$ the set $\mathcal{R} \backslash \mathcal{P}$ is a (left and right) denominator set, which means that we can localize the $\operatorname{ring} \mathcal{R}$ and any $\mathcal{R}$-module $M$ with respect to this set. The corresponding localizations are denoted by $\mathcal{R}_{\mathcal{P}}$ and $M_{\mathcal{P}}$, respectively. Analogously as in the commutative case, we define the support $\operatorname{Supp}(M)$ of $M$ as the set of primes $\mathcal{P}$ of $\mathcal{R}$ such that the localization $M_{\mathcal{P}}$ is nonzero; $\operatorname{Supp}(M)$ is a closed subset of the prime spectrum $\operatorname{Spec} \mathcal{R}$ (see Lemma 2.8). Now we are ready to state our first main result, which is proved in Section 2 in a slightly more general setting; see Theorem 2.10, and Sect. 0 for the definition of $\mathcal{V}(M)$ for a module $M$.

Theorem A. Let $\mathcal{R}$ be a ring from the class $\mathcal{K}$ and $M$ a finitely generated $\mathcal{R}$ module. Then $\operatorname{Supp}(M) \subseteq \mathcal{V}(M)$, and $\mathcal{P} M \neq M$ for any $\mathcal{P} \in \operatorname{Supp}(M)$.

Related to the above result, let us emphasize that for a commutative ring $\mathcal{R}$ and a finitely generated $\mathcal{R}$-module $M$ it is well known, and easy to see, that $\operatorname{Supp}(M)=$ 
$\mathcal{V}(M)$. But for $\mathcal{R} \in \mathcal{K}$ noncommutative the latter is in general no longer true (see Example 2.11 and Remark B.7(1)).

Now let $\mathcal{R}$ again be any left Noetherian ring, and $M$ an $\mathcal{R}$-module. For a semiprime ideal $I$ of $\mathcal{R}$ set $\overline{\mathcal{R}}:=\mathcal{R} / I$, and denote by $\Sigma(I)$ the set of all regular elements in $\overline{\mathcal{R}}$. Since Goldie's theorem ensures that $\Sigma(I)$ is a denominator set, we can localize the ring $\overline{\mathcal{R}}$ and the $\overline{\mathcal{R}}$-module $M / I M$ with respect to this set. Let $\mathcal{R}_{[I]}$ and $M_{[I]}$ denote the corresponding localizations. The localization process $\mathcal{R} \mapsto \mathcal{R}_{[I]}$ and $M \mapsto M_{[I]}$ we call the reduced localization at $I$. Now we define $\operatorname{RSupp}(M)$ as the set of primes $\mathcal{P}$ of $\mathcal{R}$ satisfying $M_{[\mathcal{P}]} \neq 0$. This set we call the reduced support of $M$. Section 3 of the paper is devoted to studying the reduced localization and in particular the reduced support. (For that purpose we define the $\mathcal{P}$-reduced functors, $\mathcal{P}$ 's being primes, as important auxiliary objects. It turns out that these functors are right exact but not necessarily left exact. It would be interesting to characterize those $\mathcal{P}$ 's for which the $\mathcal{P}$-reduced functors are exact.) Let us note that the reduced localization could be in general very hard to understand, but also sometimes it has a nice behavior; for example while working within some category of finite-dimensional modules over a left Noetherian $k$-algebra, where $k$ is a field. Besides the fact that the reduced localization and the reduced support are quite interesting for themselves, they also play an indispensable role in Section 4 for proving the results, on certain $\mathcal{U}$-modules, summarized in the next theorem; see Propositions 3.5, 4.1 and 4.6. Theorem 4.3 and Corollary 4.9

Theorem B. Suppose we have the setting as in the first paragraph of this Introduction. Let $V$ be a finitely generated $\mathfrak{n}$-module. Then:

(i) $\operatorname{RSupp}(V) \subseteq \operatorname{Supp}(V)$, with equality for $\mathfrak{n}$ abelian.

(ii) The following are equivalent:

(a) there exists (nonunique) $v \in V$ such that $s v \neq 0$ for every $s \in \mathcal{U} \backslash \mathcal{A}$;

(b) $\mathcal{A} \in \operatorname{Supp}(V)$;

(c) $\mathcal{A} \in \operatorname{RSupp}(V)$;

(d) $\mathcal{A} V \neq V$.

(iii) Suppose that $V$ is finite dimensional. Then $\mathcal{V}(V)=\operatorname{Ass} V=\operatorname{Supp}(V)$, and as a consequence we obtain that all the primes from the latter set are maximal ideals. If $V$ is moreover an $\mathfrak{a}$-module, then $\mathcal{A} \in \operatorname{Ass} V$.

(iv) Suppose that $V$ is an irreducible admissible ( $\mathfrak{g}, K$ )-module (cf. $[\mathrm{CO}, 2.3$ Thm.]). Then $V$ is finite dimensional if and only if $\mathcal{A} \in \operatorname{Ass} V$.

The above theorem has several interesting implications. First, suppose that we are interested in the general problem of nontriviality for the modules $V / \mathcal{P} V$, $\mathcal{P}$ a prime of $\mathcal{U}$; obviously, for the latter it suffices to consider only $\mathcal{P}$ 's which are moreover maximal ideals. (In particular, this is related to the question of existence of Whittaker models for Harish-Chandra modules; see [C, 6.(5)].) Then part (iv) combined with Theorem A implies that the support $\operatorname{Supp}(V)$ is in fact the object of primary interest, rather than the set of associated primes Ass $V$ (cf. Remark B.7). Next, (ii) says that the nontriviality of the module $V / \mathcal{A} V$ implies the much stronger fact that the reduced module $V_{[\mathcal{A}]}$, over $\mathcal{U}_{[\mathcal{A}]}$, is nontrivial as well (cf. Claim 2(i) of Example [3.14). Also note that the statement (a) in (ii) holds for every admissible finitely generated $(\mathfrak{g}, K)$-module; this is immediate by combining Casselman's theorem stated at the beginning and the equivalence of (a) and (d) in (ii). (For a possible approach to proving Casselman's theorem, via 
showing directly that (a) in (ii) is fulfilled for admissible finitely generated ( $\mathfrak{g}, K)$ modules, see Appendix A, where this is done in the most simple case; that is, for $G:=S L(2, \mathbb{R})$.) Finally, by (i) it follows that for $\mathfrak{n}$ abelian we have $V_{[\mathcal{P}]} \neq 0$, and so in particular $V / \mathcal{P} V \neq 0$, for every $\mathcal{P} \in \mathcal{V}(V)$ (cf. Theorem A.5).

Remark. Let $\mathfrak{n}$ be an abelian Lie algebra. Set $X:=\operatorname{Spec} U(\mathfrak{n})$. Let $\mathcal{O}_{X}$ be the structure sheaf on $X$. Also, let $M$ be a finitely generated $\mathfrak{n}$-module and $\tilde{M}$ the associated sheaf on $X$. Now, from Theorem $\mathrm{B}(\mathrm{ii})$ it follows that $\mathcal{A} M \neq M$ if and only if the stalk of $\tilde{M}$ at $\mathcal{A}$ is nontrivial; that is, if $\mathcal{A} \in \operatorname{Supp} \tilde{M}=\operatorname{Supp}(M)$. As a consequence we obtain that if $M$ is an admissible finitely generated ( $\mathfrak{g}, K)$-module (with the corresponding $\mathfrak{n}$ being abelian), then Casselman's theorem stated before is equivalent to the nonvanishing of the stalk of the sheaf $\tilde{M}$ at the augmentation ideal $\mathcal{A}$. This shows that the (geometric object) sheaf $\tilde{M}$ of $M$, and in particular its support, carry some interesting information for representation theory. In [ک̌4] we will explain how one can define the structure sheaf $\mathcal{O}_{X}$ and thus the associated sheafs of $\mathfrak{n}$-modules, for some nonabelian nilpotent Lie algebras $\mathfrak{n}$. (The construction naturally extends the classical one from commutative theory.)

The crucial reason which does not allow us to simply copy the CasselmanOsborne technique in $[\mathrm{CO}]$ for $\mathfrak{n}$ abelian to the nonabelian case is the already noted fact that for $\mathfrak{n}$ nonabelian and $V$ a finitely generated $\mathfrak{n}$-module it can happen that $\operatorname{Supp}(V)$ is strictly included in $\mathcal{V}(V)$; this is the "main obstruction" of the noncommutative theory (see Sect. 6). (Moreover, the Fact in Remark B.7 shows that possibly $\operatorname{Supp}(V)=\emptyset$ even if $V$ is also a $\mathfrak{g}$-module!) But for those $\mathfrak{g}$-modules $V$ which, when considered as $\mathfrak{n}$-modules, satisfy (1) $\operatorname{Supp}(V)=\mathcal{V}(V)$ and (2) $V$ is finitely generated, one can further pursue the Casselman-Osborne approach (cf. [CO, p.197]) by means of the next generalization of [CO, 5.1 Thm.]. (Casselman and Osborne proved their theorem about stability for associated primes; they worked under the assumption that the ideal $\mathfrak{N}$, satisfying the conditions in the theorem below, is abelian as a Lie algebra. Note here that for a finitely generated module $V$ over the enveloping algebra of any nilpotent Lie algebra, the associated Ass $V$ and the annihilator primes Annspec $V$ are the same; see Theorem 1.5. Related to the theorem which follows, recall that for Ass $V$, Annspec $V$ and the foundation primes Fnd $V$ of a finitely generated module $V$ over an arbitrary (left) Noetherian ring, we have the inclusions Ass $V \subseteq$ Annspec $V \subseteq$ Fnd $V$; see Theorem 1.3(i) and (iv), and Remark 1.4) The next main result is an immediate consequence of the more general stability result, Theorem[5.1. Let us emphasize that the proof of Theorem 5.1 strongly relies on our approach via foundation prime ideals, as developed in [Š2].

Theorem C. Let $\mathfrak{Q}$ be a finite-dimensional Lie algebra over a field $k$ of characteristic zero, and $\mathfrak{N}$ an ideal of $\mathfrak{Q}$; by $U(\mathfrak{N})$ denote the universal enveloping algebra of $\mathfrak{N}$. Let $V$ be a $\mathfrak{Q}$-module which is finitely generated as an $\mathfrak{N}$-module. Then every foundation prime of $V$, when $V$ is regarded as a $U(\mathfrak{N})$-module, is $\mathfrak{Q}$-stable for the adjoint action of $\mathfrak{Q}$ on $U(\mathfrak{N})$.

Now we will describe the organization of the paper, and also some related ideas and other facts which are in particular relevant for representation theory. For convenience of the reader and further needs we recall in Sect. 1 some definitions

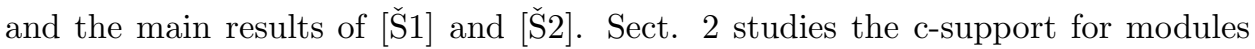


over Noetherian AR rings; the completely prime spectrum of a ring is now more natural to deal with, rather than the prime spectrum. In Sect. 3 we introduce and study the reduced localization and the reduced support for modules over (left) Noetherian rings. In Sect. 4 we apply all the previously obtained results to study $U(\mathfrak{n})$-modules, where $\mathfrak{n}$ is a nilpotent Lie algebra. In Sect. 5 we prove Theorem C and also state some of its numerous consequences (see Theorem 5.5 and Corollaries 5.6 and 5.10). Sect. 6 explains the main ideas and applications of our approach, and thus it may as well serve as the "second introduction" to the subject. Let us be more precise and in that order first describe our standard setting; that is, the setting which is the most interesting one for us. Suppose $k$ is an algebraically closed field of characteristic zero and $\mathfrak{g} \leq \mathfrak{g l}(n, k), n \in \mathbb{N}$, is a semisimple Lie algebra. Let $\mathfrak{q}$ be a (minimal) parabolic subalgebra of $\mathfrak{g}$ and let $\mathfrak{n}$ be its nilpotent radical. Suppose $Q$ is a connected closed subgroup of $G L(n, k)$ such that $\mathfrak{q}$ is its Lie algebra. Now, it turns to be natural to consider the category $\operatorname{Mod}_{\mathfrak{n} \text {-fin }}(\mathfrak{q})$ of $\mathfrak{q}$-modules which are finitely generated as $\mathfrak{n}$-modules. Our main aim is to understand the associated primes Ass $V$ and the support $\operatorname{Supp}(V)$, for modules $V$ from the above category regarded as $\mathfrak{n}$-modules; that is, as modules over the enveloping algebra $U(\mathfrak{n})$ of $\mathfrak{n}$. The first important result here is Corollary 6.8, which says that Ass $V$ is included in a pretty restricted set of prime ideals; that is, in the set $\operatorname{Spec}^{Q} U(\mathfrak{n})$ of primes of $U(\mathfrak{n})$ which are $Q$-stable for the adjoint action of $Q$ on $U(\mathfrak{n})$. Next, the very illuminating Fact 6.9 suggests that there is some "reasonable" relation between the set $\mathfrak{n}^{*} / Q$ of the (coadjoint) $Q$-orbits on the dual of $\mathfrak{n}$ and the set $\operatorname{Spec}^{Q} U(\mathfrak{n})$. And consequently, then one may hope that the latter set of primes, and thus Ass $V$ as well, is fairly small. (In Fact 6.9] we consider $\mathfrak{g}=\mathfrak{s l}(2, k)$ and $\mathfrak{g}=\mathfrak{s l}(3, k)$, respectively, where in both cases $\mathfrak{q}$ is a minimal parabolic subalgebra of $\mathfrak{g}$. Now, $\operatorname{Spec}^{Q} U(\mathfrak{n})$ has 2 and 5 elements, respectively!) Having all that we already said, the rest of Sect. [6 explains a possible approach toward Casselman's subrepresentation theorem using our techniques. (Although we do not have all the details completed yet, we strongly believe that our techniques could achieve this particular goal. But for that one should better understand the associated primes of admissible finitely generated $(\mathfrak{g}, K)$-modules.) We finish the paper with two appendices, in which we demonstrate our approach on two examples; that is, on the simple Lie algebras $\mathfrak{s l}(2)$ and $\mathfrak{s l}(3)$, respectively. Besides, this suggests how one should proceed, and what kind of results to expect, in more general situations (see Theorems A.5 and B.3, and Fact B.8).

The research presented in this paper was mainly done during the author's stay at the Massachusetts Institute of Technology, academic year 1998/99. The author expresses his sincere gratitude to Professor David Vogan for arranging this stay, and also for his enduring kindness and help.

\section{Notation and DEFinitions}

Let $\mathcal{R}$ be a ring, $A$ a subset of $\mathcal{R}, I$ an ideal of $\mathcal{R}, \mathcal{S} \subseteq \mathcal{R}$ a (left) denominator set, $M$ an $\mathcal{R}$-module, $m$ an element of $M$ and $X$ a subset of $M$. If not otherwise specified, any ring we consider has an identity, every ideal is two-sided and all modules are nontrivial left unital. Throughout we will use the following notation:

$$
\begin{aligned}
(0) & :=\text { zero-ideal of } \mathcal{R}, \\
\operatorname{ann}_{\mathcal{R}}(X) & :=\text { annihilator of } X \text { in } \mathcal{R}, \\
\operatorname{ann}_{M}(I) & :=\text { annihilator of } I \text { in } M,
\end{aligned}
$$




$$
\begin{aligned}
\langle A\rangle & :=\text { ideal of } \mathcal{R} \text { generated by } A, \\
\mathfrak{A}_{m} & :=\text { greatest ideal of } \mathcal{R} \text { which annihilates } m\left(=\operatorname{ann}_{\mathcal{R}}(\mathcal{R} m)\right), \\
\mathcal{C}(I) & :=\{r \in \mathcal{R} \mid r+I \text { is regular in } \mathcal{R} / I\} \\
\operatorname{Spec}_{\mathcal{R}} & :=\text { set of prime ideals of } \mathcal{R}, \\
\operatorname{Spec}_{c} \mathcal{R} & :=\text { set of completely prime ideals of } \mathcal{R}, \\
\operatorname{Max}_{\mathcal{R}} & :=\text { set of maximal ideals of } \mathcal{R}, \\
\mathcal{V}_{\mathcal{R}}(M) & :=\left\{\mathcal{P} \in \operatorname{Spec}_{\mathcal{R}} \mid \mathcal{P} \supseteq \operatorname{ann}_{\mathcal{R}}(M)\right\} \\
\mathcal{V}_{\mathcal{R}, c}(M) & :=\mathcal{V}_{\mathcal{R}}(M) \cap \operatorname{Spec}_{c} \mathcal{R}, \\
\mathcal{R}_{\mathcal{S}} & :=(\text { left }) \text { localization of } \mathcal{R} \text { at } \mathcal{S}, \\
M_{\mathcal{S}} & :=(\text { left) localization of } M \text { at } \mathcal{S}, \\
Q(\mathcal{R}) & :=\text { (left) quotient ring of } \mathcal{R}(\text { if exists), } \\
\operatorname{Mod}(\mathcal{R}) & :=\text { category of } \mathcal{R} \text {-modules. }
\end{aligned}
$$

Note. When there will be no ambiguity we will write just ann $(X), \mathcal{V}(M)$ and $\mathcal{V}_{c}(M)$ instead of $\operatorname{ann}_{\mathcal{R}}(X), \mathcal{V}_{\mathcal{R}}(M)$ and $\mathcal{V}_{\mathcal{R}, c}(M)$, respectively.

We consider the set $\operatorname{Spec} \mathcal{R}$ ( $\operatorname{resp} . \operatorname{Spec}_{c} \mathcal{R}$ ) equipped with the Zariski topology, and then we call it the prime (resp. completely prime) spectrum of $\mathcal{R}$; recall that the understanding of this topology means nothing else but the understanding of $\operatorname{Spec} \mathcal{U}\left(\operatorname{resp} . \operatorname{Spec}_{c} \mathcal{U}\right)$ as a lattice ordered by the inclusion relation. The closure of a subset $T$ of $\operatorname{Spec} \mathcal{R}$ (resp. $\operatorname{Spec}_{c} \mathcal{R}$ ) will be denoted by $c l(T)$. The elements of $\mathcal{R}_{\mathcal{S}}$ (resp. $M_{\mathcal{S}}$ ) will be written as the fractions $r / s$ (resp. $m / s$ ) for $r \in \mathcal{R}$ (resp. $m \in M)$ and $s \in \mathcal{S}$.

Let us emphasize that, having $\mathcal{R}, I$ and $M$ as above and a submodule $N$ of $M$, we will always denote by "bar" both the quotient homomorphisms, from $\mathcal{R}$ onto $\mathcal{R} / I$ and from $M$ onto $M / N$.

Also, we recall the definition of a centralizing sequence in a ring (see $[\mathrm{McC}]$ and [MR, 4.1.13]), and introduce some related terminology.

Definition. A sequence of elements $a_{1}, \ldots, a_{n}$ of a ring $\mathcal{R}$ is called a centralizing sequence if the following two conditions hold $(Z($. ) denotes the center of a ring):

(1) $a_{1} \in Z(\mathcal{R})$ and $\varphi_{j}\left(a_{j}\right) \in Z\left(\mathcal{R}_{j}\right)$ for $j>1$, where $\mathcal{R}_{j}:=\mathcal{R} / \sum_{i=1}^{j-1} \mathcal{R} a_{i}$ and $\varphi_{j}: \mathcal{R} \rightarrow \mathcal{R}_{j}$ is the canonical ring homomorphism; and

(2) $\sum_{i=1}^{n} \mathcal{R} a_{i} \neq \mathcal{R}$.

An ideal $I$ of $\mathcal{R}$ satisfies the $C S G$-condition if the following holds:

(CSG) $I$ has a centralizing sequence of generators.

The ring $\mathcal{R}$ satisfies the CSG-condition if every ideal of $\mathcal{R}$ satisfies this condition.

We denote by $\mathbb{C}$ and $\mathbb{R}$ the complex and real numbers, and by $\mathbb{Z}, \mathbb{N}$ and $\mathbb{Z}_{+}$the sets of integers, positive integers and nonnegative integers, respectively.

\section{Foundation PRIME IDEALS}

Let $\mathcal{R}$ be a ring and $M$ an $\mathcal{R}$-module. A prime ideal $\mathcal{P}$ of $\mathcal{R}$ such that $\mathcal{P}=$ $\operatorname{ann}(N)$ for some nonzero submodule $N$ of $M$ is called an annihilator prime for $M$. Denote

Annspec $M:=$ set of annihilator prime ideals for $M$. 
A prime $\mathcal{P}$ of $\mathcal{R}$ is associated to $M$ if there exists a nonzero submodule $N$ of $M$ such that $\mathcal{P}=\operatorname{ann}\left(N^{\prime}\right)$ for any nonzero submodule $N^{\prime}$ of $N$. Denote

$$
\text { Ass } M:=\text { set of prime ideals associated to } M \text {. }
$$

(Note that associated primes are a special kind of annihilator primes.) Further, a prime $\mathcal{P}$ is $w$-associated to $M$ if $\mathcal{P}$ is the annihilator of some nonzero cyclic submodule of $M$; that is, $\mathcal{P}=\mathfrak{A}_{m}$ for some nonzero $m \in M$. (Note that this definition is a natural generalization of the well known notion of an associated prime, from commutative theory.) Denote

$$
\text { w-Ass } M:=\text { set of prime ideals w-associated to } M \text {. }
$$

For further needs we recall the following basic result (see [ک̌2, Lemma 2.2] and [̌1, Prop. 1.5]).

Proposition and Definition 1.1. Let $\mathcal{R}$ be a ring and $M$ an $\mathcal{R}$-module. Suppose the set $\mathcal{D}(M)$ of maximal elements in w-Ass $M$ is nonempty (e.g., for $\mathcal{R}$ left Noetherian).

(i) The submodule

$$
\operatorname{fnd}(M):=\sum_{\mathcal{P} \in \mathcal{D}(M)} \operatorname{ann}_{M}(\mathcal{P})
$$

is called the foundation of $M$. The latter sum is actually direct; that is, fnd $(M)=\bigoplus_{\mathcal{P} \in \mathcal{D}(M)} \operatorname{ann}_{M}(\mathcal{P})$.

Now let $\mathcal{P} \in \mathcal{D}(M)$ be arbitrary and denote $N:=\operatorname{ann}_{M}(\mathcal{P})$.

(ii) For any nonzero $n \in N$ we have $\mathfrak{A}_{n}=\operatorname{ann}(N)$, and the latter ideal is prime.

(iii) If $N$ possesses a simple submodule, then $\operatorname{ann}(N)$ is a primitive ideal.

We also introduce the following notions and terminology (cf. [ัS2, Def. 2.3]).

Definition 1.2. A (finite or infinite) exhaustive filtration $(\mathcal{F}): 0=\mathcal{F}_{0}(M) \subset$ $\mathcal{F}_{1}(M) \subset \cdots$ of $M$, such that $\mathcal{F}_{i}(M)$ are (mutually nonequal) submodules of $M$ satisfying $\operatorname{fnd}\left(M / \mathcal{F}_{i}(M)\right)=\mathcal{F}_{i+1}(M) / \mathcal{F}_{i}(M)$ for $i=0,1, \ldots$, is called the foundation filtration of $M$. (Note that it is unique, if it exists.) The number of nonzero modules in $(\mathcal{F})$ is called the length of the foundation filtration and is denoted by ff-l $(M)$. The quotient module $\mathcal{L}^{i}(M):=\mathcal{F}_{i}(M) / \mathcal{F}_{i-1}(M)$ is called the $i$-th foundation layer. In particular, $\mathcal{L}^{1}(M)$ (resp. $\mathcal{L}^{l}(M)$, where $\left.l=\mathrm{ff}-\mathrm{l}(M)\right)$ is called the bottom (resp. top) foundation layer. Also, we define the sets of prime ideals

$\operatorname{Fnd}_{i} M:=$ set of maximal elements in w-Ass $M / \mathcal{F}_{i-1}(M)$

(note that $\operatorname{Fnd}_{1} M$ is equal to $\mathcal{D}(M)$, the latter being defined in the preceding proposition/definition), and

$$
\text { Fnd } M:=\bigcup_{i>0} \operatorname{Fnd}_{i} M
$$

The set Fnd $M$ is called the set of foundation primes of $M$ and its elements are called foundation primes of $M$. We say that the primes from $\operatorname{Fnd}_{i} M$ belong to the $i$-th foundation level. In particular, the primes from the bottom (resp. top) foundation level will be called the bottom (resp. top) level foundation primes. If $i$ is the minimal foundation level to which a foundation prime $\mathcal{P}$ of $M$ belongs, we write $\operatorname{mfl}(\mathcal{P})=i$. 
When dealing with all the above defined kinds of prime ideals, the first natural question asks how are these primes mutually related. The next theorem clarifies

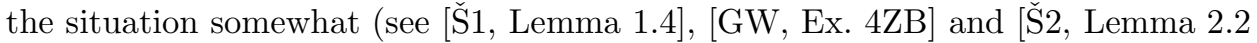
and Thm. 2.5]).

Theorem 1.3. Let $\mathcal{R}$ be a left Noetherian ring and $M$ a finitely generated $\mathcal{R}$ module. Then we have the following.

(i) A prime $\mathcal{P}$ of $\mathcal{R}$ is an annihilator prime for $M$ if and only if it is w-associated to $M$; that is, we have $\mathrm{w}-$ Ass $M=$ Annspec $M$. If $\mathcal{R}$ is in addition a commutative ring, then $\mathcal{P}$ is associated to $M$ if and only if it is w-associated to $M$.

(ii) $M$ has the finite foundation filtration.

(iii) Fnd $_{i} M$ are finite sets of mutually incomparable prime ideals.

(iv) The (finite) set Fnd $M$ contains the set $\mathrm{w}$-Ass $M$.

Note. Whenever $\mathcal{R}$ is left Noetherian and $M$ is finitely generated, we prefer in the sequel to speak about annihilator primes, rather than w-associated primes; and we use the notation Annspec $M$, rather than w-Ass $M$.

Remark 1.4. (1) Let Aff $M$ be the set of affiliated primes for $M$ (see [St]), where $\mathcal{R}$ is left Noetherian and $M$ is finitely generated. Then Annspec $M \subseteq$ Aff $M$ (see, e.g., GW, Ex. 2M]), and the sets Aff $M$ and Fnd $M$ can be different, even when

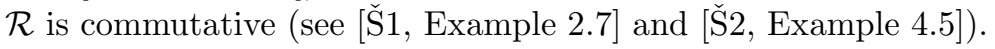

(2) Let $\mathcal{R}$ and $M$ be arbitrary. Then any prime of $\mathcal{R}$ associated to $M$ is also w-associated to $M$; that is, Ass $M \subseteq$ w-Ass $M \subseteq$ Annspec $M$ (with possible strict inclusions).

As we will see, the following nontrivial result, which deals with the problem of recognizing the annihilator primes within the set of foundation primes, is a cornerstone for our work in the present paper. It is the main result in [S2]. (Related to the second, easier, part of the theorem, cf. Remark 2.6)

Theorem 1.5. Let $\mathcal{R}$ be a left Noetherian ring satisfying the CSG-condition, $M$ a finitely generated $\mathcal{R}$-module and $\mathcal{P}$ an arbitrary foundation prime of $M$. Then the following are equivalent:

(a) $\mathcal{P} \in$ Annspec $M$;

(b) there is no $\mathcal{Q} \in$ Annspec $M$ such that $\mathcal{Q} \subset \mathcal{P}$ and $\mathrm{mfl}(\mathcal{Q})<\mathrm{mfl}(\mathcal{P})$.

If $\mathcal{R}$ is moreover a Noetherian ring, then $\mathcal{P}$ is associated to $M$ if and only if it is an annihilator prime for $M$. Therefore, now we have

$$
\text { Ass } M=\text { Annspec } M \text {. }
$$

\section{Supports AND C-Supports of MOdules over NoEtherian AR Rings}

For the terminology and results concerning the AR (Artin-Rees) property that is used below we refer to [GW, Chap. 11] and [MR, Sect. 4.2].

The following lemma is a straightforward consequence of two well known results, one due to Nouazé and Gabriel [NG], and one due to Smith [S].

Lemma 2.1. Let $\mathcal{R}$ be a Noetherian ring which satisfies the CSG-condition, and $I$ a semiprime ideal of $\mathcal{R}$. Then

(i) $\mathcal{R}$ is an AR ring;

(ii) $\mathcal{C}(I)$ is a left and right denominator set, and thus the localization $\mathcal{R}_{\mathcal{C}(I)}$ exists. 
Now we introduce a generalization of one well known and important notion from commutative theory, the support of a module.

Definition 2.2. Let $\mathcal{R}$ be a Noetherian $\mathrm{AR}$ ring and $M$ an $\mathcal{R}$-module. For a semiprime ideal $I$ of $\mathcal{R}$ write $\mathcal{R}_{I}$ and $M_{I}$ instead of $\mathcal{R}_{\mathcal{C}(I)}$ and $M_{\mathcal{C}(I)}$, respectively (cf. Lemma 2.1). The set of prime ideals

$$
\operatorname{Supp}(M):=\left\{\mathcal{P} \in \operatorname{Spec} \mathcal{R} \mid M_{\mathcal{P}} \neq 0\right\}
$$

is called the support of $M$. The set of completely prime ideals

$$
\operatorname{Supp}_{c}(M):=\operatorname{Supp}(M) \cap \operatorname{Spec}_{c} \mathcal{R}
$$

is called the c-support of $M$.

The purpose of this section is to show that the above defined support and especially c-support possess some good properties. First, having any ring $\mathcal{R}$ and an arbitrary denominator set $\mathcal{S}$, recall that the localization functor $M \mapsto M_{\mathcal{S}}$ from $\operatorname{Mod}(\mathcal{R})$ into $\operatorname{Mod}\left(\mathcal{R}_{\mathcal{S}}\right)$ is exact. Hence, we have the next basic result, which is well known when $\mathcal{R}$ is commutative.

Proposition 2.3. Let $\mathcal{R}$ be a Noetherian AR ring, $M$ an $\mathcal{R}$-module and $N$ a submodule of $M$. Then

$$
\operatorname{Supp}(M)=\operatorname{Supp}(N) \cup \operatorname{Supp}(M / N) .
$$

The next auxiliary lemma strongly relies on Theorem 1.5 .

Lemma 2.4. Let $\mathcal{R}$ be a left Noetherian (resp. Noetherian) ring, $\mathcal{P} \in \operatorname{Spec} \mathcal{R}$, and $M$ a finitely generated $\mathcal{R}$-module. Then the following two statements are equivalent:

(a) $\mathcal{P} \in \mathcal{V}(M)$;

(b) there exists $\mathcal{Q} \in$ Fnd $M$ such that $\mathcal{Q} \subseteq \mathcal{P}$. In particular, we have

$$
\mathcal{V}(M)=\operatorname{cl}(\text { Fnd } M) .
$$

If $\mathcal{R}$ moreover satisfies the CSG-condition, then the above statements are further equivalent to

(c) there exists $\mathcal{Q} \in$ Annspec $M$ (resp. $\mathcal{Q} \in$ Ass $M$ ) such that $\mathcal{Q} \subseteq \mathcal{P}$.

Proof. Let $\left(\mathcal{F}_{i}(M)\right)$ be the foundation filtration of $M$ and $l \in \mathbb{N}$ its length (see Theorem 1.3).

Suppose now that (a) holds. Then for every $i, i \leq l$, define the ideals $J_{i}:=$ $\prod_{\text {Fnd }_{i} M} \mathcal{Q}$ and set $J:=J_{1} \cdots J_{l}$. Since $J M=0$, we immediately have (b). Let us prove the opposite implication. For that purpose take $\mathcal{Q}$ as in (b). Then there exist some $j$ and $x$ such that $x \in \mathcal{F}_{j+1}(M) \backslash \mathcal{F}_{j}(M)$ and $\mathcal{Q}=\mathfrak{A}_{\bar{x}}$, where $\bar{x}:=x+\mathcal{F}_{j}(M)$. But since $\operatorname{ann}(M) x=0$, in particular we have $\operatorname{ann}(M) \subseteq \mathcal{Q}$, and so (a).

The implication (c) $\Rightarrow$ (b) follows by Theorem 1.3(iv). (Here $\mathcal{R}$ need not satisfy the CSG-condition.) For the opposite, let $\mathcal{Q}$ be as in (b) and let $\mathfrak{m}$ be a minimal element, from Fnd $M$, contained in $\mathcal{Q}$. Now we apply Theorem 1.5 , which says that $\mathfrak{m} \in \operatorname{Annspec} M($ resp. $\mathfrak{m} \in \operatorname{Ass} M)$.

The following corollary is in fact the second half of the previous lemma, rewritten in a slightly different form. 
Corollary 2.5. Let $\mathcal{R}$ be a Noetherian (resp. left Noetherian) ring which satisfies the CSG-condition, and $M$ a finitely generated $\mathcal{R}$-module. Then

$$
\mathcal{V}(M)=\operatorname{cl}(\text { Ass } M) \quad(\text { resp. } \mathcal{V}(M)=\operatorname{cl}(\text { Annspec } M)) .
$$

Remark 2.6. The first referee of this paper pointed out to us that the above corollary is essentially known, by standard Jategaonkar theory, even in more general setting. Namely, suppose that $\mathcal{R}$ is a Noetherian AR ring. Then (a) $\mathcal{R}$ satisfies the strong second layer condition and has no non-trivial links (see [J 8.1.9 Prop.]). Further, let $M$ be a finitely generated $\mathcal{R}$-module. Then (b) affiliated primes of $M$ are in the cliques of associated primes of $M$ (see [GW, Thm. 11.4]). Thus, (a) and (b) imply (c) Ass $M=$ Annspec $M=$ Aff $M$ (cf. Remark 1.4). Finally, since a suitably ordered product of affiliated primes annihilates $M$, it is clear that every prime minimal over $\operatorname{ann}(M)$ is affiliated (equiv. associated, by (c)).

The next corollary is a specialization of the above lemma.

Corollary 2.7. Let $I$ be an ideal of a left Noetherian ring $\mathcal{R}$. Consider the ring $\mathcal{R} / I$ as a left $\mathcal{R}$-module. Then the set $\mathrm{Fnd} \mathcal{R} / I$ includes all the minimal prime ideals of $\mathcal{R}$ containing $I$.

Proof. Note that $I=\operatorname{ann}(\mathcal{R} / I)$, and then use (1).

The next simple result says that $\operatorname{Supp}_{c}(M)$ is equal to the closure of its minimal elements. (Let it be said that we do not know whether the same holds for the support $\operatorname{Supp}(M)$ of $M$.

Lemma 2.8. Let $\mathcal{R}$ be a Noetherian $A R$ ring and $M$ an $\mathcal{R}$-module. If $\mathcal{P} \in$ $\operatorname{Supp}_{c}(M)$ and $\mathcal{Q} \in \operatorname{Spec}_{c} \mathcal{R}$ are such that $\mathcal{P} \subseteq \mathcal{Q}$, then $\mathcal{Q} \in \operatorname{Supp}_{c}(M)$.

Proof. Generally, if $\mathcal{R}$ is any ring and $\mathcal{S}, \mathcal{T}$ are (left) denominator sets satisfying $\mathcal{T} \subseteq \mathcal{S}$, then $M_{\mathcal{T}}=0$ implies $M_{\mathcal{S}}=0$, for an arbitrary module $M$. Hence, the lemma follows by taking $\mathcal{T}=\mathcal{C}(\mathcal{Q})$ and $\mathcal{S}=\mathcal{C}(\mathcal{P})$.

For a ring $\mathcal{R}$, a denominator set $\mathcal{S} \subseteq \mathcal{R}$, and an $\mathcal{R}$-module $M$, the set

$$
\operatorname{ass}_{M} \mathcal{S}:=\{m \in M \mid s m=0 \text { for some } s \in \mathcal{S}\}
$$

is a submodule of $M$ which is called the torsion submodule with respect to $\mathcal{S}$. The next useful proposition, which is similar to [B1, Prop. 2.2.4] with the only difference that we allow $\mathcal{R}$ to be a noncommutative ring, relates the localization and the torsion submodule. Although this result is well known (see, e.g., MR, 2.1.17 Prop.]), for the sake of completeness and further needs we provide a detailed proof inspired by [B1].

Proposition 2.9. $M_{\mathcal{S}}=0$ if and only if $M=\operatorname{ass}_{M} \mathcal{S}$. More precisely, for elements $s \in \mathcal{S}$ and $m \in M$, the fraction $\mathrm{m} / \mathrm{s}$ equals zero in $M_{\mathcal{S}}$ if and only if there exists $s^{\prime} \in \mathcal{S}$ such that $s^{\prime} m$ equals zero in $M$.

Proof. Suppose that $s^{\prime} m=0$ for some $s^{\prime} \in \mathcal{S}$. Then $1 / s^{\prime} \mathrm{m} / s=s^{\prime} m / s^{\prime} s s^{\prime}=0 / 1$.

Let us prove the opposite implication. For any $t \in \mathcal{S}$, denote the set $\{r / t \mid r \in \mathcal{R}\}$ by $\mathcal{R}_{t}$. As in [B1], conclude that there exist $t \in \mathcal{S}$ such that $m / 1=0 / 1$ in the $\mathbb{Z}$ module $\mathcal{R}_{t} \otimes_{\mathcal{R}} M$. (Here we use the analog of [B2, Prop. 3.1.8] for noncommutative rings, and [D, 3.6.3].) Denote by $\mathcal{B}$ the kernel of the right $\mathcal{R}$-module homomorphism $r \mapsto r / t$ from $\mathcal{R}$ onto $\mathcal{R}_{t}$. We can write the element $t m=\sum_{i=1}^{n} b_{i} m_{i}$, where $b_{i} \in \mathcal{B}$ and $m_{i} \in M$. Since $b_{1} / t=0 / 1$, there exist $\alpha_{1}, \sigma_{1} \in \mathcal{R}$ such that $\alpha_{1} b_{1}=0$ and 
$\sigma_{1}=\alpha_{1} t \in \mathcal{S}$. It follows that $\sigma_{1} m=\sum_{i=2}^{n} c_{i} m_{i}$, where $c_{i}:=\alpha_{1} b_{i}$ are elements from $\mathcal{R}_{\sigma_{1}}$. By continuing this construction, in at most $n$ steps we find $s^{\prime} \in \mathcal{S}$ such that $s^{\prime} m=0$.

The following theorem is the main result of this section.

Theorem 2.10. Let $\mathcal{R}$ be a Noetherian $A R$ ring and $M$ a finitely generated $\mathcal{R}$ module. Then

(i) $\mathcal{P} M \neq M$ for every $\mathcal{P} \in \operatorname{Supp}_{c}(M)$;

(ii) $\operatorname{Supp}_{c}(M) \subseteq \mathcal{V}_{c}(M)$.

Proof. (i) Suppose the contrary; that is, that $\mathcal{P} M=M$ for some $\mathcal{P}$. Then we immediately have $\mathcal{P}^{k} M=M$, for every $k \in \mathbb{N}$. Now take an arbitrary $m \in M$ and consider the cyclic module $C:=\mathcal{R} m$. Since $\mathcal{R}$ is a Noetherian AR ring, there exists some $l \in \mathbb{N}$ satisfying $\mathcal{P}^{l} M \cap C \subseteq \mathcal{P} C$, which obviously implies $\mathcal{P} C=C$. In particular, there exists some $p \in \mathcal{P}$ such that $p m=m$. Therefore $m \in \operatorname{ass}_{M} \mathcal{C}(\mathcal{P})$, and hence $M_{\mathcal{P}}=0$ (see Proposition 2.9), which is a contradiction.

(ii) Take any ideal $\mathcal{P} \in \operatorname{Supp}_{c}(M)$. Then choose some $m \in M$ such that $s m \neq 0$ for every $s \in \mathcal{C}(\mathcal{P})$. Now let $J$ be the ideal defined in the proof of Lemma 2.4 Then $J m=0$, wherefrom we immediately deduce that $J \cap \mathcal{C}(\mathcal{P})=\emptyset$. Therefore we have $J \subseteq \mathcal{P}$, and so $\mathcal{Q} \subseteq \mathcal{P}$ for some ideal $\mathcal{Q} \in$ Fnd $M$. It remains to apply Lemma 2.4.

The following example of Dixmier's (see [CO, p.198], and also Appendix B) shows that, for $\mathcal{R}$ noncommutative, in (ii) of the previous theorem we can have strict inclusion.

Example 2.11. Let $\mathfrak{n}$ be the three-dimensional Heisenberg Lie algebra, say over $\mathbb{C}$; that is, $\mathfrak{n}:=\mathbb{C} x \oplus \mathbb{C} y \oplus \mathbb{C} z$ with the commutator relations $[x, y]=z$ and $[x, z]=[y, z]=0$. Consider the ring $\mathcal{U}:=U(\mathfrak{n})$, the left ideal $L:=\mathcal{U}(1+x)$, and the $\mathcal{U}$-module $V:=\mathcal{U} / L$. Also, let $\mathcal{A}$ be the augmentation ideal of $\mathcal{U}$.

Now, the zero-ideal is the only annihilator (equiv. associated) prime of $V$, which means that $\mathcal{V}(V)=\operatorname{Spec} \mathcal{U}$. At the same time we have the following. (Recall that now $\operatorname{Supp}_{c}()=.\operatorname{Supp}($.$) .)$

Claim. $\mathcal{A} \notin \operatorname{Supp}(V)$.

[By denoting $B:=\left\{z^{i} y^{j}(1+x)^{k} \mid i, j, k \in \mathbb{Z}_{+}\right\}$it is easy to see that $B$ is a vector space basis for $\mathcal{U}$. Therefore, any nonzero $\omega \in V$ can be written as $\omega=\sum_{i, j} \gamma_{i j} z^{i} y^{j}+L$, for some $\gamma_{i j} \in \mathbb{C}$. By induction on $k \in \mathbb{N}$ it immediately follows that

$$
(1+x)^{k} y^{j} \equiv(1+x)^{k-1} y^{j}(1+x) \quad(\bmod L) \equiv 0 \quad(\bmod L),
$$

for every $j \in \mathbb{Z}_{+}$. Hence, choosing $n \in \mathbb{N}$ satisfying $n>j$, for every $j$ (and some $i$ ) such that $\gamma_{i j} \neq 0$, we deduce $(1+x)^{n} \omega=0$. Since $(1+x)^{n} \notin \mathcal{A}$, the claim follows.]

(The above proof is quite direct. We could also observe that for the cyclic vector $c:=1+L$ of $V$ we have $x c=-c$, which implies $\mathcal{A} V=V$. Now the claim follows by Theorem 4.3, below.)

The following simple observation follows immediately from [B1, Cor. 2.2.3].

Lemma 2.12. Let $\mathcal{R}$ be a commutative ring, $M$ a finitely generated $\mathcal{R}$-module and $I$ a proper ideal of $\mathcal{R}$ satisfying $I \supseteq \operatorname{ann}(M)$. Then $I M \neq M$. 


\section{REDUCED LOCALIZATION AND REDUCED SUPPORT}

In the previous section we considered the localizations $\mathcal{R}_{I}$ and $M_{I}$ for $\mathcal{R}$ a Noetherian AR ring, $M$ an $\mathcal{R}$-module and $I$ a semiprime ideal of $\mathcal{R}$. As it is well known, when $\mathcal{R}$ is assumed only to be (left, right) Noetherian, this construction will often be impossible (see [MR, Chap. 4]). Besides, Goldie's theorem [MR, Thm. 2.3.6] and the notion of the reduced rank [MR, Sect. 4.1] inspire the following definition.

Definition 3.1. Let $\mathcal{R}$ be a left Noetherian ring, $M$ an $\mathcal{R}$-module and $I$ a semiprime ideal of $\mathcal{R}$. Let $\Sigma(I)$ denote the set of regular elements in $\mathcal{R} / I$. (Note that $\Sigma(I)=\varphi(\mathcal{C}(I))$, where $\varphi: \mathcal{R} \rightarrow \mathcal{R} / I$ is the canonical ring homomorphism.) Define

$$
\mathcal{R}_{[I]}:=Q(\mathcal{R} / I)
$$

and

$$
M_{[I]}:=(M / I M)_{\Sigma(I)}
$$

We say that the ring $\mathcal{R}_{[I]}$ and the module $M_{[I]}$ are obtained by reduced localization at $I$. (Note that by Goldie's theorem we know that the reduced localization indeed exists at any semiprime ideal of $\mathcal{R}$.)

In Definition 2.2 we defined the support and the c-support for modules over Noetherian AR rings. In accordance with the previous definition we introduce the following.

Definition 3.2. Let $\mathcal{R}$ be a left Noetherian ring and $M$ an $\mathcal{R}$-module. The set of prime ideals

$$
\operatorname{RSupp}(M):=\left\{\mathcal{P} \in \operatorname{Spec} \mathcal{R} \mid M_{[\mathcal{P}]} \neq 0\right\}
$$

is called the reduced support of $M$.

Remark 3.3. Let $\mathcal{R}$ be a left Noetherian $\operatorname{ring}, \mathcal{P} \in \operatorname{Spec} \mathcal{R}$, and $M$ an $\mathcal{R}$-module. Recall that the rank of $M$ with respect to $\mathcal{P}$ is defined as $\rho_{\mathcal{P}}(M):=\operatorname{udim} M_{[\mathcal{P}]}$ (see [MR, 4.6.7]). Hence, we could define the reduced support of $M$ equivalently as

$$
\operatorname{RSupp}(M):=\left\{\mathcal{P} \in \operatorname{Spec} \mathcal{R} \mid \rho_{\mathcal{P}}(M) \neq 0\right\} .
$$

For a prime $\mathcal{P}$ of a left Noetherian ring $\mathcal{R}$ we define the functor

$$
\begin{array}{rlr}
\Re_{\mathcal{P}}: \operatorname{Mod}(\mathcal{R}) & \longrightarrow & \operatorname{Mod}\left(\mathcal{R}_{[\mathcal{P}]}\right), \\
\Re_{\mathcal{P}}(M):=M_{[\mathcal{P}]} & \text { and } & \Re_{\mathcal{P}}(\varphi):=\varphi_{[\mathcal{P}]},
\end{array}
$$

where we put

$$
\varphi_{[\mathcal{P}]}(\bar{m} / \sigma):=\overline{\varphi(m)} / \sigma \quad \text { for } \sigma \in \Sigma(\mathcal{P}), m \in M .
$$

The map $\varphi_{[\mathcal{P}]}$ we call the reduced homomorphism associated to $\varphi$.

The following well known result (see, e.g., [D, Prop.'s 3.6 .15 and 3.6.17]) is included for convenience of the reader.

Proposition 3.4. Let $\mathcal{R}$ be a Noetherian ring and $\mathcal{S}$ a left denominator set. Then the maps $\mathcal{P} \mapsto \mathcal{P}_{\mathcal{S}}$ and $\mathcal{P} \mapsto \mathcal{P} \cap \mathcal{R}$ between the sets $\{\mathcal{P} \in \operatorname{Spec} \mathcal{R} \mid \mathcal{P} \cap \mathcal{S}=\emptyset\}$ and Spec $\mathcal{R}_{\mathcal{S}}$ are inverses of each other. Further, for any prime $\mathcal{P}$ of $\mathcal{R}, \mathcal{P} \cap \mathcal{S}=\emptyset$, we have

$$
Q\left(\mathcal{R}_{\mathcal{S}} / \mathcal{P}_{\mathcal{S}}\right)=Q(\mathcal{R} / \mathcal{P})
$$


Proposition and Definition 3.5. Let $\mathcal{R}$ be a left Noetherian ring.

(i) For any $\mathcal{P} \in \operatorname{Spec} \mathcal{R}, \Re_{\mathcal{P}}$ is a well defined covariant right-exact functor which we call the $\mathcal{P}$-reduced functor.

(ii) Suppose that $\mathcal{R}$ is moreover a Noetherian AR ring, and let $M$ be an $\mathcal{R}$-module. Then

$$
\operatorname{RSupp}(M) \cap \operatorname{Spec}_{c} \mathcal{R} \subseteq \operatorname{Supp}_{c}(M) .
$$

In particular, if $\mathcal{R}$ is commutative and $M$ is finitely generated, then in (4) the sets are equal; that is,

$$
\operatorname{RSupp}(M)=\operatorname{Supp}(M)
$$

Proof. (i) Let $M$ and $N$ be $\mathcal{R}$-modules and $\varphi \in \operatorname{Hom}_{\mathcal{R}}(M, N)$. The proof that the map $\varphi_{[\mathcal{P}]}$ is well defined and $\mathcal{R}_{[\mathcal{P}]}$-linear is straightforward.

Let

$$
M^{\prime} \stackrel{\varphi}{\longrightarrow} M \stackrel{\psi}{\longrightarrow} M^{\prime \prime} \rightarrow 0
$$

be an exact sequence of $\mathcal{R}$-modules. We have to show that the sequence of $\mathcal{R}_{[\mathcal{P}]^{-}}$ modules

$$
M_{[\mathcal{P}]}^{\prime} \stackrel{\varphi_{[\mathcal{P}]}}{\longrightarrow} M_{[\mathcal{P}]} \stackrel{\psi_{[\mathcal{P}]}}{\longrightarrow} M_{[\mathcal{P}]}^{\prime \prime} \longrightarrow 0
$$

is exact, as well. Now, the composition of $\varphi_{[\mathcal{P}]}$ and $\psi_{[\mathcal{P}]}$ is obviously trivial, and so $\operatorname{im} \varphi_{[\mathcal{P}]} \subseteq \operatorname{ker} \psi_{[\mathcal{P}]}$. Let us prove the opposite inclusion. For that purpose suppose that $\psi_{[\mathcal{P}]}(\bar{m} / \sigma)=0$. By Proposition 2.9 there exists an element $s \in \mathcal{R}$ satisfying $\bar{s} \in \Sigma(\mathcal{P})$ and $\psi(s m)=\sum_{i} p_{i} m_{i}^{\prime \prime}$ for some $p_{i} \in \mathcal{P}, m_{i}^{\prime \prime} \in M^{\prime \prime}$. Since $\psi$ is an epimorphism, there exist $m_{i} \in M$, for every $i$, such that $\psi\left(s m-\sum_{i} p_{i} m_{i}\right)=0$. The exactness of the given sequence provides us with $m^{\prime} \in M^{\prime}$ satisfying $\varphi\left(m^{\prime}\right)=$ $s m-\sum_{i} p_{i} m_{i}$. Now we have $\varphi_{[\mathcal{P}]}\left(\overline{m^{\prime}} / \bar{s} \sigma\right)=\bar{m} / \sigma$, and this is what we had to prove. The claim that $\psi_{[\mathcal{P}]}$ is an epimorphism is obvious.

(ii) Suppose that $M_{\mathcal{P}}=0$ for some $\mathcal{P} \in \operatorname{Spec}_{c} \mathcal{R}$. By Proposition 2.9 this supposition is equivalent to the statement

$$
\forall m \in M, \exists \sigma(m) \in \mathcal{C}(\mathcal{P}) \text { such that } \sigma(m) m=0 .
$$

The latter implies $\overline{\sigma(m)} \bar{m}=0$, and at the same time we clearly have $\overline{\sigma(m)} \in \Sigma(\mathcal{P})$. Using Proposition 2.9] once again, we get $M_{[\mathcal{P}]}=0$. This proves the inclusion (4).

Now let $\mathcal{R}$ be commutative, $M$ finitely generated and $\mathcal{P} \in \operatorname{Supp}(M)$. Denote the domain $\mathcal{R} / \mathcal{P}$ by $\mathcal{D}$. Since $\mathcal{V}(M)=\operatorname{Supp}(M)$, we have $\mathcal{P} \supseteq \operatorname{ann}(M)$. Using this fact and Lemma 2.12, we see that $E:=M / \mathcal{P} M$ is a nonzero $\mathcal{D}$-module. Now by [D, Lemma 2.6.3] there exists a nonzero element $f \in \mathcal{D}$ such that the localized module $E_{f}$ is a free, and finitely generated, $\mathcal{D}_{f}$-module. Take some basis $\left(e_{i}\right)$ of $E_{f}$. If we denote the Ore set $\left(\mathcal{D}_{f}\right) \backslash\{0\}$ by $\mathcal{S}$, then by Proposition 3.4 it follows that $\left(\mathcal{D}_{f}\right)_{\mathcal{S}}=\mathcal{R}_{[\mathcal{P}]}$. Hence, it is easy to conclude that $\left(e_{i}\right)$ is a basis of the vector space $\left(E_{f}\right)_{\mathcal{S}}=M_{[\mathcal{P}]}$. This proves that $\mathcal{P} \in \operatorname{RSupp}(M)$, which finishes the proof.

Remark 3.6. The above proof of (i) is quite direct. Also, we could proceed in the following way. Let $I$ be an arbitrary ideal of a ring $\mathcal{R}$. Then

$$
\begin{aligned}
& r_{I}: \operatorname{Mod}(\mathcal{R}) \longrightarrow \operatorname{Mod}(\mathcal{R} / I), \\
& r_{I}(M):=M / I M \quad \text { and } \quad r_{I}(\varphi)(\bar{m}):=\overline{\varphi(m)},
\end{aligned}
$$

is a well defined covariant right-exact functor. (The proof is along the same lines as for reduced functors, but easier.) Now it remains to observe that for any $I$ such 
that $\Sigma(I)$ is left Ore, the functor $\Re_{I}$ is the composition of $r_{I}$ and the tensoring functor $\mathcal{R}_{\Sigma(I)} \otimes_{\mathcal{R}}-$.

The following simple fact will be very useful in the sequel.

Corollary 3.7. Let $\mathcal{R}$ be a left Noetherian ring, $M$ an $\mathcal{R}$-module and $N$ a submodule of $M$. Then

$$
\operatorname{RSupp}(M / N) \subseteq \operatorname{RSupp}(M) .
$$

Proof. This follows directly from (i) of the previous proposition.

The reduced functors are not necessarily exact, even on short exact sequences of finitely generated modules over commutative Noetherian domains. To see this, consider the following example. (This is [Š2, Example 4.5] specialized for $n=2$; see also [̌́1, Example 2.7].)

Example 3.8. Let $k$ be an algebraically closed field and $\mathcal{A}:=k\left[X_{1}, X_{2}\right]$ the polynomial ring in two variables. Define the ideals $\mathcal{I}:=\left\langle X_{1} X_{2}\right\rangle, \mathcal{P}_{1}:=\left\langle X_{2}\right\rangle, \mathcal{P}_{2}:=\left\langle X_{1}\right\rangle$ and $\mathcal{Q}:=\left\langle X_{1}, X_{2}\right\rangle$. Also, define the $\mathcal{A}$-module $M:=\mathcal{A} / \mathcal{I}$ and its submodules $N^{1}:=\mathcal{P}_{2} / \mathcal{I}$ and $N^{2}:=\mathcal{P}_{1} / \mathcal{I}$.

Now consider the embedding $\imath: N^{1} \hookrightarrow M$ and the reduced homomorphism $\imath_{[\mathcal{P}]}: N_{[\mathcal{P}]}^{1} \rightarrow M_{[\mathcal{P}]}$, for $\mathcal{P} \in \operatorname{Spec} \mathcal{A}$. First observe that $N_{[\mathcal{P}]}^{1} \neq 0$ if and only if $\mathcal{P}_{1} \subseteq \mathcal{P}$ (use (15) and the facts that $\mathcal{V}\left(N^{1}\right)=\operatorname{Supp}\left(N^{1}\right)$ and $\mathcal{P}_{1}=\operatorname{ann}\left(N^{1}\right)$ ). In particular, $N_{\left[\mathcal{P}_{1}\right]}^{1}$ and $N_{[\mathcal{Q}]}^{1}$ are nonzero modules. But, also, we have

Claim. (i) $\imath_{\left[\mathcal{P}_{1}\right]}$ is injective.

(ii) $\imath_{[\mathcal{Q}]}$ is trivial.

[Let us, for example, show (i). For that purpose take an arbitrary $n:=\alpha X_{1}+\mathcal{I}$ from $N^{1}, \alpha \in \mathcal{A}$, and assume that (use (3))

$$
\imath_{\left[\mathcal{P}_{1}\right]}\left(\left(n+\mathcal{P}_{1} N^{1}\right) /\left(a+\mathcal{P}_{1}\right)\right)=\left(n+\mathcal{P}_{1} M\right) /\left(a+\mathcal{P}_{1}\right)=0, \quad a \notin \mathcal{P}_{1} .
$$

This means that there exists some $s \notin \mathcal{P}_{1}$ such that $s n \in N^{2}$ (observe that $\mathcal{P}_{1} M=$ $\left.N^{2}\right)$. Hence, there exists some $p \in \mathcal{P}_{1}$ such that $s \alpha X_{1}-p \in \mathcal{I}$. Now we easily deduce $\alpha \in \mathcal{P}_{1}$, which further means that $n \in \mathcal{P}_{1} N^{1}$. So, $\operatorname{ker} \imath_{\left[\mathcal{P}_{1}\right]}=0$, as we had to show.]

In the next few simple lemmas some interesting facts concerning the reduced localization and reduced functors are brought to our attention.

Lemma 3.9. Let $\mathcal{R}$ be a left Noetherian prime ring, $\Sigma:=\Sigma((0))$ the set of regular elements in $\mathcal{R}$ and $M$ a finitely generated unfaithful $\mathcal{R}$-module. Then $M$ is torsion with respect to $\Sigma$; that is, $M=\operatorname{ass}_{M} \Sigma$ (see (20).

Proof. Combine Lemma 3.8(i) and Corollary 3.9 of [ $\underline{\mathrm{S} 2}$.

Lemma 3.10. Let $\mathcal{R}$ be a left Noetherian ring and $M$ a finitely generated $\mathcal{R}$ module. Then

$$
\operatorname{RSupp}(M) \subseteq \mathcal{V}(M) .
$$

Proof. Suppose that $\mathcal{P} \in \mathrm{RSupp}(M)$ and $\operatorname{ann}(M) \nsubseteq \mathcal{P}$. Define the quotient ring $\overline{\mathcal{R}}:=\mathcal{R} / \mathcal{P}$ and consider the $\overline{\mathcal{R}}$-module $\bar{M}:=M / \mathcal{P} M$. Then $\bar{M}$ is unfaithful, since the nonzero ideal $(\operatorname{ann}(M)+\mathcal{P}) / \mathcal{P}$ of $\overline{\mathcal{R}}$ annihilates $\bar{M}$. Hence, by Lemma 3.9, it follows that $M_{[\mathcal{P}]}=0$; a contradiction. 
Lemma 3.11. Let $M$ be a module over a left Noetherian ring $\mathcal{R}$.

(i) If $M$ is a semisimple module (cf. Remark 3.15 below), then

$$
\operatorname{RSupp}(M) \subseteq \mathrm{w}-\operatorname{Ass} M .
$$

(ii) If $M$ is a simple module and $\operatorname{RSupp}(M)=\{\mathcal{P}\}$, where $\mathcal{P}=\operatorname{ann}(M)$, then the module $M_{[\mathcal{P}]}$ is simple as well. Furthermore, if $N$ is an arbitrary $\mathcal{R}$-module and $\varphi \in \operatorname{Hom}_{\mathcal{R}}(M, N)$, then the reduced homomorphism $\varphi_{[\mathcal{P}]}$ is either trivial or injective.

Proof. For an arbitrary prime ideal $\mathcal{Q}$ of $\mathcal{R}$ and any family $\left(N^{i}\right)_{I}$ of submodules of $M$ satisfying $M=\bigoplus_{I} N^{i}$, we have

$$
M_{[\mathcal{Q}]}=\bigoplus_{I} N_{[\mathcal{Q}]}^{i},
$$

and hence

$$
\operatorname{RSupp}(M)=\bigcup_{I} \operatorname{RSupp}\left(N^{i}\right)
$$

(i) Clearly, by the above observation we can assume that $M$ is simple. Then denote $\mathcal{P}:=\operatorname{ann}(M)$. Now, if $\mathcal{Q} \in \operatorname{RSupp}(M)$ then obviously $\mathcal{Q} \subseteq \mathcal{P}$. It remains to apply Lemma 3.10 .

(ii) Obviously, if the module $M_{[\mathcal{P}]}$ is nontrivial then it is simple. Hence, by Schur's lemma, the claim on $\varphi_{[\mathcal{P}]}$ immediately follows.

Lemma 3.12. Let $\mathcal{R}$ be a left Noetherian $k$-algebra, $k$ a field, and $M$ an $\mathcal{R}$-module which possesses a finite foundation filtration (for example, $M$ finitely generated). Denote by $T$ the top foundation layer of $M$. Let $\mathcal{P}$ be a top level foundation prime of $M$, and assume that moreover one of the following two possibilities is fulfilled: either

(1) the submodule $\operatorname{ann}_{T}(\mathcal{P})$ has a simple submodule, which is a finite dimensional $k$-vector space, as a direct summand; or

(2) $\mathcal{P} \in \operatorname{Max} \mathcal{R}$ and $\operatorname{ann}_{T}(\mathcal{P})$ has a simple quotient which as a $k$-vector space is finite dimensional.

Then $\mathcal{P} \in \operatorname{RSupp}(M)$.

Proof. Case (2). Let $E$ be a simple quotient of $\operatorname{ann}_{T}(\mathcal{P}), \operatorname{dim}_{k} E<\infty$. Using the fact that $\mathcal{P} \in \operatorname{Max} \mathcal{R}$, it immediately follows that $\mathcal{P}=\operatorname{ann}(E)$. Now take any vector space basis $B$ of $E$. Then there exists some $e_{0} \in B$ such that $\sigma e_{0} \neq 0$ for every $\sigma \in \mathcal{C}(\mathcal{P})$; which means that $E_{[\mathcal{P}]} \neq 0$. (Indeed. Otherwise, by using the Ore condition several times we could find some $\omega \in \mathcal{C}(\mathcal{P})$ satisfying $\omega e=0$ for every $e \in B$, and hence it would follow that $\omega E=0$; which is impossible.) To finish the proof one must observe that $E$ can be also considered as a quotient of $M$, and then apply Corollary 3.7

Case (1). Write $\operatorname{ann}_{T}(\mathcal{P})=E \oplus V$, for some module $V$ and a simple finite dimensional $E$, and note that $\mathcal{P}=\operatorname{ann}(E)$. Then proceed as in Case (2).

The following result will provide an essential step in the proof of Theorem 4.3 .

Proposition 3.13. Let $\mathcal{R}$ be a Noetherian $A R k$-algebra, $k$ a field, and $M$ a finitely generated $\mathcal{R}$-module. Let $\mathcal{P}$ be an ideal of $\mathcal{R}$ which satisfies the following three conditions: 
(I) $\mathcal{P} \in \operatorname{Supp}_{c}(M)$,

(II) $\operatorname{dim}_{k} \mathcal{R} / \mathcal{P}<\infty$

(III) $\mathcal{P} \in \operatorname{Max} \mathcal{R}$.

Then $\mathcal{P} \in \operatorname{RSupp}(M)$.

Proof. By (I) and Theorem 2.10(i) we have that $V:=M / \mathcal{P} M$ is a nontrivial $k$ vector space. By (II) and the fact that $M$ is finitely generated we conclude that $\operatorname{dim}_{k} V<\infty$. Further, let $N$ be a submodule of $M$ which contains $\mathcal{P} M$ and is such that $E:=M / N$ is a simple $\mathcal{R}$-module. Using (III) we obtain that $\mathcal{P}=\operatorname{ann}(E)$. Now, by Lemma 3.12 we have $\mathcal{P} \in \operatorname{RSupp}(E)$ (put $E$ instead of $M$ in the lemma), and so the proposition follows by Corollary 3.7 .

From our considerations in this and the next section it will follow that the reduced localization has a nice behavior when we deal with some category of finitedimensional modules over a (left) Noetherian $k$-algebra, $k$ a field. But in the following interesting example (see [V1 Example 1.27]) we show that quite different experiences occur while dealing with infinite-dimensional modules.

First we agree on our notation. Let $\mathfrak{g}$ be a semisimple Lie algebra, say over $\mathbb{C}$. Fix a Borel subalgebra $\mathfrak{b}$ in $\mathfrak{g}$, and $\mathfrak{h}$ a Cartan subalgebra contained in $\mathfrak{b}$. By $\mathfrak{n}$ denote the nilpotent radical of $\mathfrak{b}$, and by $\mathfrak{n}^{-}$the corresponding nilpotent radical opposite to $\mathfrak{n}$. Let $R(\mathfrak{g}, \mathfrak{h})$ be the root system with the positive roots determined by $\mathfrak{b}$, and $\rho$ the half-sum of all positive roots. By $\mathbb{C}_{\vartheta}, \vartheta \in \mathfrak{h}^{*}$, denote the one-dimensional $\mathfrak{b}$-module defined on $\mathbb{C}$ such that $\mathfrak{n} .1=0$ and $\mathfrak{h} .1=\vartheta(\mathfrak{h})$. Then for $\lambda \in \mathfrak{h}^{*}$ consider the Verma module $M(\lambda):=U(\mathfrak{g}) \otimes_{U(\mathfrak{b})} \mathbb{C}_{\lambda-\rho}$, its largest submodule $M^{1}(\lambda)$ and the (unique) simple quotient module $L(\lambda):=M(\lambda) / M^{1}(\lambda)$ (see [D, Chap. 7]).

Example 3.14. Let $\mathfrak{g}:=\mathfrak{s l}(2, \mathbb{C})$, and let $\{x, y, h\}$ be its standard basis, so that, in accordance with the above notation, we have $\mathfrak{h}=\mathbb{C} h, \mathfrak{n}=\mathbb{C} x, \mathfrak{b}=\mathfrak{h} \oplus \mathfrak{n}$ and $\mathfrak{n}^{-}=\mathbb{C} y$. Fix $\alpha \in \mathfrak{h}^{*}$ such that $\alpha(h)=2$. Then $R(\mathfrak{g}, \mathfrak{h})=\{-\alpha, \alpha\}$ and $\rho=\alpha / 2$. For a $\lambda \in \mathfrak{h}^{*}$ we say that $\lambda$ corresponds to $z \in \mathbb{C}$, and write $\lambda \leftrightarrow z$, if $\lambda(h)=z$. Now, for every $z \in \mathbb{C}$ define the Verma module $V_{z}:=M(\lambda)$, where $\lambda \leftrightarrow z$. Further, by $\Omega$ denote the Casimir element $4 x y+h^{2}-2 h$ of $U(\mathfrak{g})$, and then define the ideals $I_{z}:=\left\langle\Omega-\left(z^{2}-1\right)\right\rangle, z \in \mathbb{C}$. Although the following simple fact is certainly well known, for convenience of the reader and further needs we give a direct elementary proof.

Claim 1. $I_{z}=\operatorname{ann}\left(V_{z}\right), z \in \mathbb{C}$.

[It is well known that, as a $U\left(\mathfrak{n}^{-}\right)$-module, $V_{z}$ is generated by $\left\{y^{i} \otimes 1\right\}_{i \geq 0}$. Now it is straightforward to see that $\left\langle\Omega-\left(z^{2}-1\right)\right\rangle \cdot y^{i} \otimes 1=0$ for every $i$, which proves the inclusion from left to right. For the opposite, take some $F \in \operatorname{ann}\left(V_{z}\right)$ and decompose it, with respect to the PBW-basis associated to the chosen standard basis, as $F=\sum_{i, j, k} \gamma_{i j k} x^{i} y^{j} h^{k}, \gamma_{i j k} \in \mathbb{C}$. Since (assume $i, j>0$ ) we have

$$
x^{i} y^{j} h^{k}=\frac{1}{4} x^{i-1} \Omega y^{j-1} h^{k}-\frac{1}{4} x^{i-1}\left(h^{2}-2 h\right) y^{j-1} h^{k},
$$

and also $h y=y h-2 h$, it is clear that we can write the monomials of $F$ as $x^{i} y^{j} h^{k}=$ $w+x^{i-1} y^{j-1} q(h)$, for some $w \in I_{z}$ and some polynomial $q \in \mathbb{C}[T]$. This means that

$$
F=w+\sum_{i \geq 1} a_{i j} x^{i} h^{j}+\sum_{k \geq 1} b_{k l} y^{k} h^{l}+P(h)
$$


for some $w \in I_{z}, P \in \mathbb{C}[T]$ and coefficients $a_{i j}, b_{k l} \in \mathbb{C}$. Moreover, we can order $F$ by the powers of $x$ and $y$ as

$$
F=w+\sum_{i=1}^{e} x^{i} q_{i}(h)+\sum_{j=1}^{f} y^{j} p_{j}(h)+P(h),
$$

where $q_{i}, p_{j}, P \in \mathbb{C}[T]$ and $e, f \in \mathbb{N}$. By induction it is now easy to see that for every $n \in \mathbb{N}$ we have $h^{m} \cdot y^{n} \otimes 1=(z-2 n-1)^{m} y^{n} \otimes 1, m \in \mathbb{N}$, and so $P(h) \cdot y^{n} \otimes 1=P(z-2 n-1) y^{n} \otimes 1$. Therefore, since $F \cdot y^{n} \otimes 1=0$ for every $n$, it follows that $P(z-2 n-1)=0$ for every $n$, and thus finally $P \equiv 0$. Analogously, we see that $q_{i} \equiv 0$ and $p_{j} \equiv 0$ for all $i, j$. So, $F=w \in I_{z}$; and the claim is proved.]

Now fix an arbitrary $z \in \mathbb{C}$ and define the module $M:=V_{z}$ and the ideals $\mathcal{P}:=I_{z}, \mathcal{Q}:=\operatorname{ann}(L(\lambda))$, where $\lambda \leftrightarrow z$. With the fact proven in Claim 1 we have

Claim 2. (i) $M_{[\mathcal{P}]}=0$.

(ii) $\operatorname{RSupp}(M)= \begin{cases}\emptyset & \text { if } z \notin \mathbb{N} \\ \{\mathcal{Q}\} & \text { if } z \in \mathbb{N} \text {. }\end{cases}$

[(i) Take any $m \in M$ and decompose it as $m=\sum_{i=0}^{n} c_{i} y^{i} \otimes 1$, with $n \in \mathbb{N}$ and $c_{i} \in \mathbb{C}$. Now, by easy induction it immediately follows that $x^{n+1} \cdot m=0$. Finally, using Proposition 2.9 and the well known fact that $\mathcal{P}$ is a completely prime ideal, the proposed equality follows.

(ii) If $z \notin \mathbb{N}$, which is equivalent to the fact that $M$ is simple (see $[\mathrm{D}$, Thm. 7.6.24]), then from (i) and Lemma 3.11(i) it immediately follows that the reduced support of $M$ is void. Now, if $z \in \mathbb{N}$ then $L(\lambda)$ is finite dimensional, and thus $\mathcal{Q} \in \operatorname{RSupp}(M)$. Also, if $\mathcal{Q}^{\prime} \in \operatorname{RSupp}(M)$ then $\mathcal{P} \subset \mathcal{Q}^{\prime}$, by Lemma 3.10 and (i) again. Finally, from the well known result of Nouazé and Gabriel [NG] that every chain of prime ideals of $U(\mathfrak{g})$ is of length $\leq 2$, we deduce that $\mathcal{Q}^{\prime}$ is a maximal ideal. But then clearly $\mathcal{Q}^{\prime}=\mathcal{Q}$, which finishes the proof of the claim.]

Remark 3.15. Having the general setting as in the paragraph preceding the above example, assume $\lambda$ to be chosen so that $L(\lambda)$ is finite dimensional; that is, $\lambda-\rho$ is a dominant weight. Set $\mathcal{P}:=\operatorname{ann}(M(\lambda))$ and $\mathcal{Q}:=\operatorname{ann}(L(\lambda))$. Then $\mathcal{P} \subset \mathcal{Q}$. Now, by Lemma 3.12 and Corollary 3.7 we know that $\mathcal{Q} \in \operatorname{RSupp}(M(\lambda))$, while Annspec $M(\lambda)=\{\mathcal{P}\}$ by the lemma given below. This fact together with Claim 2 of Example 3.14 shows that the set of annihilator primes and the reduced support are, in general, incomparable.

Lemma 3.16. Let $M$ be a Verma module. Then $\operatorname{fnd}(M)=M$ and

$$
\text { Ass } M=\text { Annspec } M=\text { Fnd } M=\operatorname{Fnd}_{1} M=\{\operatorname{ann}(M)\} \text {. }
$$

Proof. See [Š1, Lemma 2.2 and Cor. 2.3].

\section{Associated primes of $U(\mathfrak{n})$-MOdules}

Throughout this section, if not otherwise said, we fix the following notation: $k$ is a field of characteristic zero, $\mathfrak{n}$ is a finite-dimensional nilpotent Lie algebra over $k, \mathcal{U}$ is the universal enveloping algebra of $\mathfrak{n}$ and $\mathcal{A}:=\mathcal{U} \mathfrak{n}$ is the augmentation ideal of $\mathcal{U}$. Recall that now $\operatorname{Spec} \mathcal{U}=\operatorname{Spec}_{c} \mathcal{U}$; therefore, $\mathcal{V}(V)=\mathcal{V}_{c}(V)$ and $\operatorname{Supp}(V)=\operatorname{Supp}_{c}(V)$, for every $\mathcal{U}$-module $V$. Also, for a finitely generated $\mathcal{U}$ module $V$ we have Ass $V=\operatorname{Annspec} V(=$ w-Ass $V)$. 
Somewhat roughly speaking, the following interesting result shows that the finitedimensional $\mathcal{U}$-modules can be treated similarly to the finitely generated modules over commutative Noetherian rings.

Proposition 4.1. Let $V$ be a finite-dimensional $\mathcal{U}$-module. Then

$$
\mathcal{V}(V)=\operatorname{Ass} V=\operatorname{Supp}(V)
$$

and, in particular, $\mathcal{V}(V) \subseteq \operatorname{Max} \mathcal{U}$.

Proof. First we prove that

$$
\text { Ass } V=\text { Fnd } V \subseteq \operatorname{Max} \mathcal{U} .
$$

To see this, take an arbitrary prime $\mathcal{P} \in$ Fnd $V$. Since $V$ is finite dimensional, the ideal $\mathcal{P}$ is primitive (by Proposition [1.1(iii)). By [D, 4.7.4 Prop.] we know that every primitive ideal of $\mathcal{U}$ is moreover maximal, and so we have the inclusion Fnd $V \subseteq \operatorname{Max} \mathcal{U}$. Hence, using Theorem 1.5 we immediately obtain Ass $V=$ Fnd $V$. (Note that both (7) and the argument given for its proof hold even if $V$ is an Artinian module.)

Now let $\mathcal{P} \in \operatorname{Ass} V$. Then consider any simple submodule $E$ of $\operatorname{ann}_{V}(\mathcal{P})$. By Proposition 1.1(ii) we know that $\mathcal{P}=\mathfrak{A}_{e}$ for every nonzero $e \in E$. Thus, using Lemmas 3.11(i) and 3.12 we clearly have $\operatorname{RSupp}(E)=\operatorname{Ass} E=\{\mathcal{P}\}$. Hence, by (41) and Proposition 2.3 we deduce that $\mathcal{P} \in \operatorname{Supp}(V)$. In that way we have proved

$$
\text { Ass } V \subseteq \operatorname{Supp}(V),
$$

but moreover we have

$$
\mathcal{V}(V)=\operatorname{cl}(\operatorname{Ass} V)=\operatorname{Ass} V \subseteq \operatorname{cl}(\operatorname{Supp}(V))=\operatorname{Supp}(V) \subseteq \mathcal{V}(V),
$$

the latter by Corollary 2.5, (7), (8), Lemma 2.8 and Theorem 2.10(ii), respectively. Thus we have (6), which finishes the proof of the proposition.

For further purposes let us note the following simple fact which is an obvious consequence of the above proof of (77).

Corollary 4.2. Let $E$ be a simple $\mathcal{U}$-module. Then

$$
\mathcal{V}(E)=\operatorname{Ass} E=\{\operatorname{ann}(E)\} .
$$

As we already noted, our understanding of the associated primes becomes more complicated while dealing with infinite-dimensional $\mathcal{U}$-modules. But for a certain prime ideal, namely $\mathcal{A}$, which seems to be an associated prime for many interesting (finite-dimensional; cf. Corollary 4.9 below) $\mathcal{U}$-modules, something can be said. The following is the main result of this section.

Theorem 4.3. Let $V$ be a finitely generated $\mathcal{U}$-module. Then the following four statements are equivalent:

(a) there exists $v \in V$ such that $s v \neq 0$ for every $s \in \mathcal{U} \backslash \mathcal{A}$;

(b) $\mathcal{A} \in \operatorname{Supp}(V)$;

(c) $\mathcal{A} \in \operatorname{RSupp}(V)$;

(d) $\mathcal{A} V \neq V$.

If $V$ is moreover an Artinian module, then the above (a)-(d) are further equivalent to the following "geometric condition" (cf. Remark [6.12(3)):

(e) $\mathcal{A} \in \mathcal{V}(V)$. 
Proof. (a) $\Leftrightarrow$ (b) This is obvious from the definition of the support and Proposition 2.9. (b) $\Rightarrow$ (c) Take $\mathcal{P}=\mathcal{A}$ in Proposition 3.13. (c) $\Rightarrow$ (b) This follows from (4). (b) $\Rightarrow$ (d) This follows by Theorem 2.10(i). (d) $\Rightarrow$ (a) Let $v \in V \backslash \mathcal{A} V$ be arbitrary. We claim that then $s v \neq 0$ for every $s \notin \mathcal{A}$. To see this suppose the contrary, and observe that any $s$ can be written as $s=\gamma+\alpha$ for some nonzero $\gamma \in k$ and $\alpha \in \mathcal{A}$. But then $v=-\frac{1}{\gamma} \alpha v$, which in particular means that $v \in \mathcal{A} V$; a contradiction.

Now let $V$ be an Artinian module; then it has a finite composition series, say $0=V_{0} \subset V_{1} \subset \cdots \subset V_{n}=V$. Denote the (simple) modules $Y_{i}:=V_{i} / V_{i-1}$ for $i=$ $1, \ldots, n$, and suppose that (e) holds. Since $\mathcal{V}(V)=\bigcup_{i=1}^{n} \mathcal{V}\left(Y_{i}\right)$, there exists some $i_{0}$ such that $\mathcal{A} \in \mathcal{V}\left(Y_{i_{0}}\right)$. Hence, by Corollary 4.2 we have $\mathcal{A} Y_{i_{0}}=0$, and therefore by the equivalence of (b) and (d) applied to $Y_{i_{0}}$ we have $\mathcal{A} \in \operatorname{Supp}\left(Y_{i_{0}}\right)$. Finally, Proposition 2.3 gives $\mathcal{A} \in \operatorname{Supp}(V)$, which finishes the proof of the implication $(\mathrm{e}) \Rightarrow(\mathrm{b})$. By Theorem 2.10(ii) we have $(\mathrm{b}) \Rightarrow(\mathrm{e})$; note how now $V$ need not be Artinian.

Corollary 4.4. Let $V$ be a finitely generated $\mathcal{U}$-module and suppose that $\mathcal{A}$ is an associated prime of $V$. Then $\mathcal{A} V \neq V$.

Proof. Since $\mathcal{A}$ is an associated (equiv. w-associated) prime of $V$, there exists some $v \in V$ such that $\mathcal{A}=\mathfrak{A}_{v}$. But then statement (a) of the above theorem is fulfilled for $v$.

We can further strengthen the last corollary. Namely, we have the following:

Corollary 4.5. Let $V$ be a finitely generated $\mathcal{U}$-module and suppose that there exists a (nontrivial) subquotient $Y$ of $V$ having $\mathcal{A}$ as an associated prime. Then $\mathcal{A} V \neq V$.

Proof. By the previous corollary we have $\mathcal{A} Y \neq Y$, which is by Theorem 4.3 further equivalent to $\mathcal{A} \in \operatorname{Supp}(Y)$. Using Proposition 2.3 and Theorem 4.3 again, we deduce that $\mathcal{A} V \neq V$.

For the rest of this section, we will work under the following setting. Let $\mathfrak{g}$ be a complex semisimple Lie algebra and $\mathfrak{k} \oplus \mathfrak{a} \oplus \mathfrak{n}$ its Iwasawa decomposition (standard notation). Define the Lie subalgebra $\mathfrak{f}:=\mathfrak{a} \oplus \mathfrak{n}$, and let $\mathcal{U}$ and $\mathcal{A}$ be as before.

Proposition 4.6. Let $V$ be a finite-dimensional f-module. Then $\mathcal{A}$ is an associated prime of $V$, where $V$ is considered as a $\mathcal{U}$-module.

Proof. Let $\Phi$ be the restricted root system of $\mathfrak{g}$ with respect to $\mathfrak{a}$, and $\Phi^{+}$the (positive) roots such that $\mathfrak{n}=\sum_{\varphi \in \Phi^{+}} \mathfrak{g}_{\varphi}$; the $\mathfrak{g}_{\varphi}$ 's are the usual root subspaces. Let $a \in \mathfrak{a}$ be such that $\varphi(a)>0$ for every $\varphi \in \Phi^{+}$, and consider the operator $A$ on $V, A(v):=a v$ for $v \in V$. Now we have the following two possibilities: either (a) $A(V)=0$, or (b) $A(V) \neq 0$. If (a) then it immediately follows that $\gamma_{\varphi} V=0$, for every $\varphi \in \Phi^{+}$and an arbitrary $\gamma_{\varphi} \in \mathfrak{g}_{\varphi}$. Hence, we have $\mathcal{A} V=0$ and thus, moreover, Fnd $V=\{\mathcal{A}\}$. If (b), let $\lambda_{1}, \ldots, \lambda_{j}$ be the eigenvalue set of $A$. Assume that, for example, $\lambda_{1}+\varphi(a)$ is not an eigenvalue of $A$, for every $\varphi \in \Phi^{+}$. Then consider an arbitrary $\lambda_{1}$-eigenvector $w$. We claim that $\gamma_{\varphi} w=0$ for any $\gamma_{\varphi}, \gamma_{\varphi}$ being as before. To prove this decompose $\gamma_{\varphi} w=\sum_{i=1}^{j} v_{i}$, with $v_{i}$ from the $\lambda_{i}$-eigenspace. Acting by $A$ on the last equality, we immediately obtain

$$
\left(\lambda_{1}+\varphi(a)\right) \sum_{i=1}^{j} v_{i}=\sum_{i=1}^{j} \lambda_{i} v_{i}
$$


which obviously implies $v_{i}=0$ for every $i$, and so $\gamma_{\varphi} w=0$ as claimed. Thus $\mathcal{A} \in$ Annspec $V$, which by means of Proposition 4.1 finishes the proof of the proposition.

Corollary 4.7. Let $V$ be an $\mathfrak{f}$-module which is finitely generated as an $\mathfrak{n}$-module, and suppose that the following condition is fulfilled:

(*) there exists a proper $\mathfrak{f}$-submodule $W$ of finite codimension in $V$.

Then $\mathcal{A} V \neq V$.

Proof. Denote $Y:=V / W$. By Proposition 4.6 we have $\mathcal{A} \in \operatorname{Ass} Y$, and so by Corollary $4.4 \mathcal{A} Y \neq Y$. Now we apply the same argument as in the proof of Corollary 4.5.

Remark 4.8. Stafford and Wallach ([SW, Sect. 4]; see also Remark B.7 (1)) showed that for $\mathfrak{g}:=\mathfrak{s} l(3, k)$ there exists a family $\left\{M_{\alpha} \mid \alpha \in k\right\}$ of $\mathfrak{g}$-modules such that each $M_{\alpha}$, when considered as an $\mathfrak{n}$-module, has one generator and it satisfies $\mathcal{A} M_{\alpha}=$ $M_{\alpha}$. This means that one cannot remove condition $(*)$ from the above corollary. Also, if $V$ is an admissible finitely generated $(\mathfrak{g}, K)$-module, then condition $(*)$ is fulfilled; namely, Casselman's theorem stated in the Introduction gives that, for example, $W=\mathcal{A} V$ satisfies $(*)$. Note that if one could prove $(*)$ for $(\mathfrak{g}, K)$ modules, independently, that would yield another algebraic proof of Casselman's subrepresentation theorem.

The next easy result shows that Proposition 4.6 cannot be extended to the case of infinite-dimensional modules.

Corollary 4.9. Let $V$ be an irreducible admissible $(\mathfrak{g}, K)$-module. Then $V$ is finite dimensional if and only if $\mathcal{A}$ is an associated prime of $V$, where $V$ is considered as a $\mathcal{U}$-module.

Proof. If $\mathcal{A} \in \operatorname{Ass} V$, then $\mathcal{A}=\mathfrak{A}_{v}$ for some nonzero vector $v \in V$, and thus in particular $v$ is an $\mathfrak{n}$-invariant vector. By [CO, 2.6 Cor.] it follows that $V$ is finite dimensional.

\section{StABILITY RESUlt FOR FOUNDATION PRIMES}

Let $\mathcal{R}$ be an arbitrary ring and $\partial$ some derivation of $\mathcal{R}$. Also, let $M$ be any $\mathcal{R}$-module. Recall that a derivation of $M$ over $\partial$ is an additive map $\Delta: M \rightarrow M$ which moreover satisfies

$$
\Delta(r m)=\partial(r) m+r \Delta(m) \quad \text { for all } r \in \mathcal{R}, m \in M .
$$

The main purpose of this section is to establish the following generalization of one algebraic result, given by Casselman and Osborne in case the ring $\mathcal{R}$ is commutative [CO, 4.5 Thm.]; in fact, they proved that every associated prime of $M$ is $\partial$-stable, for $\mathcal{R}, M$ and $\partial$ as in the theorem below. (Recall that a ring is called equicharacteristic if it contains a field.)

Theorem 5.1. Let $\mathcal{R}$ be a left Noetherian equicharacteristic ring of characteristic zero, and $M$ a finitely generated $\mathcal{R}$-module. Suppose that we have a pair $(\partial, \Delta)$ consisting of a derivation $\partial$ of $\mathcal{R}$ and a derivation $\Delta$ of $M, \Delta$ over $\partial$. Then every foundation prime ideal of $M$ is $\partial$-stable. 
We will prove this theorem by combining some Casselman-Osborne ideas with the technique developed in $\underline{\mathrm{S} 2}$. Let us emphasize how we essentially use a nice "structural picture" of the organization of foundation primes by foundation levels. First, we need the following crucial lemma (cf. [CO, 4.3 Prop.]).

Lemma 5.2. Let $\mathcal{R}$ be a ring and $\mathcal{P}$ an arbitrary ideal of $\mathcal{R}$. Define the additive group

$$
\mathcal{P}^{\partial}:=\sum_{\substack{0 \leq i \leq j \\ j>0}} \partial^{i}\left(\mathcal{P}^{j}\right)
$$

(we take $\left.\partial^{0}\left(\mathcal{P}^{j}\right)=\mathcal{P}^{j}\right)$. Then:

(i) $\mathcal{P}^{\partial}$ is an ideal of $\mathcal{R}$.

(ii) Suppose that $\mathcal{R}$ is an equicharacteristic ring, $\operatorname{char}(\mathcal{R})=0$, and $\mathcal{P}$ is a prime ideal. Moreover, suppose that there exists a $\partial$-stable ideal $I$ of $\mathcal{R}$ such that $\mathcal{P}^{\mu} \subseteq I \subseteq \mathcal{P}$, for some $\mu \in \mathbb{N}$. Then

$$
\mathcal{P}^{\partial}=\mathcal{P} \text {. }
$$

Proof. (i) By induction on $i$ we immediately prove the inclusion

$$
\mathcal{R} \partial^{i}\left(\mathcal{P}^{j}\right) \subseteq \sum_{0 \leq k \leq i} \partial^{k}\left(\mathcal{P}^{j}\right),
$$

for an arbitrary $j$. This implies $\mathcal{R} \mathcal{P}^{\partial} \subseteq \mathcal{P}^{\partial}$; that is, $\mathcal{P}^{\partial}$ is a left ideal of $\mathcal{R}$. Clearly, $\mathcal{P}^{\partial}$ is a right ideal, too.

(ii) Using Leibniz's formula we prove, by induction on $i$, that

$$
\partial^{i}\left(\mathcal{P}^{j}\right) \equiv \begin{cases}(\partial \mathcal{P})^{i} \quad(\bmod \mathcal{P}) & \text { for } i=j \\ 0 \quad(\bmod \mathcal{P}) & \text { for } i<j\end{cases}
$$

Now for every $1 \leq j \leq \mu$ choose some $i_{j} \in \mathbb{N}$ arbitrarily and set $\alpha:=\sum_{j=1}^{\mu} i_{j}$. Since $\mathcal{P}^{m} \subseteq I$ for any $m \geq \mu$, then in particular $\mathcal{P}^{\alpha} \subseteq I$, and thus

$$
\partial^{\alpha}\left(\mathcal{P}^{\alpha}\right) \equiv 0 \quad(\bmod \mathcal{P})
$$

Using (10) and the latter fact, it follows that

$$
\prod_{j=1}^{\mu} \partial^{i_{j}}\left(\mathcal{P}^{i_{j}}\right) \equiv(\partial \mathcal{P})^{\alpha} \quad(\bmod \mathcal{P}) \equiv 0 \quad(\bmod \mathcal{P})
$$

Hence, by the congruence (use (10) again)

$$
\left(\mathcal{P}^{\partial}\right)^{\mu} \equiv \sum_{\left(i_{1}, \ldots, i_{\mu}\right) \in \mathbb{N}^{\mu}}\left(\prod_{j=1}^{\mu} \partial^{i_{j}}\left(\mathcal{P}^{i_{j}}\right)\right) \quad(\bmod \mathcal{P}),
$$

we have $\left(\mathcal{P}^{\partial}\right)^{\mu} \subseteq \mathcal{P}$, and then the inclusion $\mathcal{P}^{\partial} \subseteq \mathcal{P}$ follows by the primeness of $\mathcal{P}$. Thus, obviously, we have (9).

We also need the following general, but easy, result.

Lemma 5.3. Let $\mathcal{R}$ be a ring and $\partial$ its derivation. Let $M$ be an $\mathcal{R}$-module having a derivation $\Delta$, over $\partial$. Suppose that $N$ is a $\Delta$-stable submodule of $M$ (that is, $\Delta(n) \in N$ for every $n \in N)$. Then $\bar{\Delta}, \bar{\Delta}(\bar{m}):=\overline{\Delta(m)}$, defines a derivation of the quotient module $M / N, \bar{\Delta}$ over $\partial$.

Proof. Straightforward; left to the reader. 
Proof of Theorem [5.1. Let us denote by $\mathcal{E}(M)$ the set of minimal modules in the set $\left\{\operatorname{ann}_{M}\left(\mathfrak{A}_{m}\right) \mid 0 \neq m \in M\right\}$ of annihilators in $M$. By the duality between the annihilators in $\mathcal{R}$ and $M, \mathcal{E}(M)$ corresponds to $\operatorname{Fnd}_{1} M$ and so in particular we have fnd $(M)=\bigoplus_{N \in \mathcal{E}(M)} N$ (cf. Proposition and Definition 1.1 and Definition 1.2).

Now, fix an arbitrary $N \in \mathcal{E}(M)$ and any nonzero $n \in N$. Then $\mathcal{P}:=\mathfrak{A}_{n}$ is a prime from $\mathrm{Fnd}_{1} M$, and

$$
N=\operatorname{ann}_{M}(\mathcal{P}) .
$$

Further, define the module

$$
M\{\mathcal{P}\}:=\left\{m \in M \mid \mathcal{P}^{\alpha} m=0 \text { for some } \alpha \in \mathbb{N}\right\} ;
$$

observe that $N \leq M\{\mathcal{P}\}$. As in $[\mathrm{CO}$ we conclude that $M\{\mathcal{P}\}$ is $\Delta$-stable. Also, for every $\nu \in \mathbb{N}$ define the module

$$
M_{\nu}\{\mathcal{P}\}:=\left\{m \in M \mid \mathcal{P}^{\nu} m=0\right\} .
$$

Since $M$, and thus $M\{\mathcal{P}\}$ also, are Noetherian modules, there exists $\mu \in \mathbb{N}$ such that $M\{\mathcal{P}\}=M_{\mu}\{\mathcal{P}\}$; that is, $\mathcal{P}^{\mu} M\{\mathcal{P}\}=0$. By setting $I:=\operatorname{ann}(M\{\mathcal{P}\})$ we immediately deduce the inclusions $\mathcal{P}^{\mu} \subseteq I \subseteq \mathcal{P}$. (Note that $I$ is $\partial$-stable.) From Lemma 5.2 it follows that $\mathcal{P}$ is $\partial$-stable.

Clearly, by the previous lemma, to finish the proof of the theorem it remains to show that the foundation $\operatorname{fnd}(M)$ is a $\Delta$-stable submodule of $M$. To see this it is sufficient to prove that even the module $N$ is $\Delta$-stable. Now, let us assume that $\Delta(n)$ is a nonzero element. Since $\mathcal{P}$ is a $\partial$-stable ideal, we have $\mathcal{P} \Delta(n)=0$, and so in particular $\mathcal{P} \subseteq \mathfrak{A}_{\Delta(n)}$. In fact, by the maximality of $\mathcal{P}$ the last inclusion is moreover an equality. Thus we deduce $\Delta(n) \in N$ (use (11)). This finishes the proof of the theorem.

Proof of Theorem $C$. For every $\lambda \in \mathfrak{Q}$ let $\partial_{\lambda}$ be the unique derivation of $U(\mathfrak{N})$ which extends the derivation $\operatorname{ad}_{\mathfrak{N}} \lambda$ on $\mathfrak{N}$ (see (14) below). Also, define $\Delta_{\lambda}$ by $\Delta_{\lambda}(x):=\lambda x, x \in V$. By induction on the standard filtration of $U(\mathfrak{N})$ one can easily see that $\Delta_{\lambda}$ is a derivation of $V$ over $\partial_{\lambda}$. Now apply Theorem 5.1 to the pair $\left(\partial_{\lambda}, \Delta_{\lambda}\right)$.

We will finish this section with some observations which are easy consequences of Theorem 5.1 and/or Theorem C.

Taking into account Corollary 2.7, it follows that the next corollary extends a well known stability result for primes minimal over an ideal (see, e.g., $\mathrm{D}$, 3.3.3 Lemma]).

Corollary 5.4. Let $\mathcal{R}$ be a left Noetherian equicharacteristic ring of characteristic zero, $\partial$ a derivation of $\mathcal{R}$ and $I$ a $\partial$-stable ideal of $\mathcal{R}$. Then every foundation prime ideal of the (left) $\mathcal{R}$-module $\mathcal{R} / I$ is $\partial$-stable.

Proof. By Lemma 5.3 we know that $\bar{\partial}$ is a derivation of $\mathcal{R} / I, \bar{\partial}$ over $\partial$. Hence, the corollary follows by Theorem 5.1 .

As one more application of Theorem $\mathrm{C}$ we also quote the next result, whose proof strongly relies on the latter (cf. [D, §3.3]); for a proof and some further

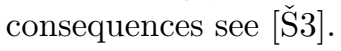


Theorem 5.5. Let $\mathfrak{Q}, \mathfrak{N}$ and $V$ be as in Theorem $C$. Write $V_{1}$ for $V$ considered as an $\mathfrak{N}$-module, and let $\operatorname{ind}\left(V_{1}, \mathfrak{Q}\right)$ be the $\mathfrak{Q}$-module induced from $V_{1}$ (in the usual sense). Then:

(i) $U(\mathfrak{Q}) \mathcal{P}$ is a prime ideal of $U(\mathfrak{Q})$, for every foundation prime $\mathcal{P}$ of $V_{1}$.

(ii) The map

$$
\begin{aligned}
\Theta_{V}: \operatorname{Annspec}_{U(\mathfrak{N})} V_{1} & \longrightarrow \operatorname{Annspec}_{U(\mathfrak{Q})} \operatorname{ind}\left(V_{1}, \mathfrak{Q}\right), \\
\mathcal{P} & \longmapsto U(\mathfrak{Q}) \mathcal{P},
\end{aligned}
$$

is a "going up" map; that is, $\Theta_{V}(\mathcal{P}) \cap U(\mathfrak{N})=\mathcal{P}$ for every annihilator prime $\mathcal{P}$ of $V_{1}$.

In general, having a Lie algebra $\mathfrak{Q}$ and its subalgebra $\mathfrak{N}$ it is very interesting to study the restrictions of $\mathfrak{Q}$-modules to $\mathfrak{N}$. The next result deals with this problem in a certain special case (cf. [D, 5.6.2]).

Corollary 5.6. Let $\mathfrak{Q}, \mathfrak{N}$ and $k$ be as in Theorem $C$, and suppose that $\mathfrak{N}$ is moreover nilpotent as a Lie algebra. Let $(\pi, V)$ be an irreducible representation of $\mathfrak{Q}$ such that

(1) $V$ is finitely generated as an $\mathfrak{N}$-module, and

(2) the restriction $\pi_{\mid \mathfrak{N}}$ possesses an absolutely irreducible subrepresentation $(\sigma, E)$.

Then we have the following.

(i) $\pi_{\mid \mathfrak{N}}$ is a multiple of $\sigma$.

(ii) If moreover the field $k$ is algebraically closed and $V$ is finite dimensional, then $\pi_{\mid[\mathfrak{N}, \mathfrak{Q}]} \equiv 0$.

Proof. (i) Let $\mathcal{P}$ be the annihilator of $E$, in $U(\mathfrak{N})$. Then $\mathcal{P}$ is a $\mathfrak{Q}$-stable primitive ideal (by Theorem $\mathrm{C}$ ) and therefore the stabilizer of $\sigma$ in $\mathfrak{Q}$ is equal to $\mathfrak{Q}$ (by $\mathrm{D}$. 5.3.3(ii) Prop.]). Now the claim follows by [D, 5.4.1 Prop.].

(ii) By (i), and using the fact that an irreducible finite-dimensional representation of a nilpotent Lie algebra (over an algebraically closed field) must be onedimensional, there exists a basis $v_{1}, \ldots, v_{n}$ of $V$ such that, for $V_{i}:=k v_{i}$, every $V_{i}$ is an $\mathfrak{N}$-module isomorphic to $E$, and $V=\bigoplus V_{i}$. Thus, there exists an $f \in \mathfrak{N}^{*}$, $f_{\mid[\mathfrak{N}, \mathfrak{N}]} \equiv 0$, satisfying $\mathfrak{N} v_{i}=f(\mathfrak{N}) v_{i}$ for every $i$. Now take any $\lambda \in \mathfrak{Q}$ and $\alpha \in \mathfrak{N}$. Then $\lambda v_{j}=\sum x_{i}^{(j)} v_{i}$, for some $x_{i}^{(j)} \in k$. Hence, by acting with $\alpha$ on the last equality, we immediately obtain $[\alpha, \lambda] v_{j}=0$. Since $j$ was also arbitrarily chosen, it follows that $[\mathfrak{N}, \mathfrak{Q}] V=0$, which is what we had to show.

Remark 5.7. Note that the claim (ii) of the previous corollary in particular implies the following well known fact. Let $\mathfrak{g}$ be a semisimple Lie algebra and $\mathfrak{q}$ a parabolic subalgebra with the Levi decomposition $\mathfrak{q}=\mathfrak{l} \oplus \mathfrak{u}$, where $\mathfrak{l}$ and $\mathfrak{u}$ are the reductive and nilpotent part, respectively. Then every irreducible finite-dimensional representation of $\mathfrak{q}$ is trivial on $\mathfrak{u}$. (Take $\mathfrak{Q}=\mathfrak{q}$ and $\mathfrak{N}=\mathfrak{u}$ in the corollary.)

Let $k$ be an algebraically closed field, $\operatorname{char}(k)=0$. Suppose $\mathfrak{Q}$ is an algebraic Lie algebra in $\mathfrak{g l}(n, k), n \in \mathbb{N}$. Let $Q$ be a connected closed subgroup of $G L(n, k)$ such that $\mathfrak{Q}$ is the Lie algebra of $Q$. Consider the adjoint representation

$$
\begin{aligned}
& \text { Ad }: Q \longrightarrow \operatorname{Aut}(\mathfrak{Q}), \\
& \operatorname{Ad} q(X):=q X q^{-1} \quad \text { for } q \in Q, X \in \mathfrak{Q}
\end{aligned}
$$


$(\operatorname{Aut}(\mathfrak{Q}):=$ group of automorphisms of $\mathfrak{Q})$. Suppose now that $\mathfrak{N}$ is an arbitrary ideal of $\mathfrak{Q}$. Then we have the following easy lemma (cf. the proof of Proposition 5.9 given below).

Lemma 5.8. The Lie algebra $\mathfrak{N}$ is $Q$-stable for the adjoint representation; that is, Ad $q(\mathfrak{N}) \subseteq \mathfrak{N}$ for every $q \in Q$.

By means of this lemma we may consider the restriction of the representation Ad to $\mathfrak{N}$; that is, the representation

$$
\begin{aligned}
& \operatorname{Ad}_{\mathfrak{N}}: Q \longrightarrow \operatorname{Aut}(\mathfrak{N}), \\
& \operatorname{Ad}_{\mathfrak{N}} q:=(\operatorname{Ad} q)_{\mid \mathfrak{N}} \quad \text { for } q \in Q .
\end{aligned}
$$

Further, denote by $\mathcal{U}$ the enveloping algebra of $\mathfrak{N}$, and consider the extension of $\operatorname{Ad}_{\mathfrak{N}}$ to $\mathcal{U}$; that is, the representation

$$
\operatorname{Ad}_{\mathcal{U}}: Q \longrightarrow \operatorname{Aut}(\mathcal{U})
$$

where $\operatorname{Ad}_{\mathcal{U}} q$, for $q \in Q$, is the unique extension of the automorphism $\operatorname{Ad}_{\mathfrak{N}} q$ of $\mathfrak{N}$ to an automorphism of $\mathcal{U}$. Recall that the differential of $\operatorname{Ad}_{\mathcal{U}}$ is the homomorphism of Lie algebras

$$
\begin{aligned}
& \operatorname{ad}_{\mathcal{U}}: \mathfrak{Q} \longrightarrow \operatorname{Der}(\mathcal{U}), \\
& \operatorname{ad}_{\mathcal{U}} X(u):=X u-u X \quad \text { for } X \in \mathfrak{Q}, u \in \mathcal{U}
\end{aligned}
$$

$(\operatorname{Der}(\mathcal{U}):=$ Lie algebra of derivations of $\mathcal{U})$. Now we can state the following generalization of one well known stability result (see, e.g., [D, 2.4.17 Prop.]); for convenience of the reader we include an argument.

Proposition 5.9. Suppose we have the above setting. Let $I$ be an ideal of $\mathcal{U}$. Then $I$ is $Q$-stable for the representation $\mathrm{Ad}_{\mathcal{U}}$ if and only if $I$ is $\mathfrak{Q}$-stable for the representation $\operatorname{ad}_{\mathcal{U}}$; that is, $\operatorname{Ad}_{\mathcal{U}} q(I) \subseteq I$ for every $q \in Q$ if and only if $\operatorname{ad}_{\mathcal{U}} X(I) \subseteq$ I for every $X \in \mathfrak{Q}$.

Proof. Suppose $I$ is $\mathfrak{Q}$-stable. Let $\left(\mathcal{U}_{n}\right)_{n \geq 0}$ be the canonical filtration of $\mathcal{U}$, and consider the rational representations (use Lemma 5.8)

$$
\begin{aligned}
& \pi_{n}: Q \longrightarrow G L\left(\mathcal{U}_{n}\right), \\
& \pi_{n}(q):=\left(\operatorname{Ad}_{\mathcal{U}} q\right)_{\mid \mathcal{U}_{n}} \quad \text { for } q \in Q ;
\end{aligned}
$$

$\pi_{n}$ is the restriction of the representation $\operatorname{Ad}_{\mathcal{U}}$ to $\mathcal{U}_{n}$. Now, denote $I_{n}:=I \cap \mathcal{U}_{n}$. It will suffice to show that moreover $I_{n}$ is $Q$-stable for the representation $\pi_{n}$, for every $n$. To see this, define the algebraic groups $G_{n}:=\left\{q \in Q \mid \pi_{n}(q)\left(I_{n}\right) \subseteq I_{n}\right\}$. Then $\mathfrak{g}_{n}:=\left\{X \in \mathfrak{Q} \mid \mathrm{d} \pi_{n}(X)\left(I_{n}\right) \subseteq I_{n}\right\}$ is the Lie algebra of $G_{n}$; here $\mathrm{d} \pi_{n}$ is the differential of $\pi_{n}$, that is, $\mathrm{d} \pi_{n}(X)=\operatorname{ad}_{\mathcal{U}} X_{\mid \mathcal{U}_{n}}$ for $X \in \mathfrak{Q}$. Since obviously $\mathfrak{g}_{n}=\mathfrak{Q}$ for every $n$, it follows that $G_{n}=Q$ for every $n$. Thus, $I$ is $Q$-stable. The opposite implication is now clear.

Let $\mathfrak{Q}, Q$ and $\mathfrak{N}$ be as above. Denote by $\mathfrak{N}^{*}$ the dual of the $k$-vector space $\mathfrak{N}$. The contragredient representation of $\mathrm{Ad}_{\mathfrak{N}}$ is called the coadjoint representation of $Q$ on $\mathfrak{N}^{*}$. By means of it we have the coadjoint action of $Q$ on $\mathfrak{N}^{*}$ :

$$
\begin{aligned}
Q \times \mathfrak{N}^{*} & \longrightarrow \mathfrak{N}^{*}, \\
(q, \varphi) & \longmapsto q \cdot \varphi:=\varphi \circ \operatorname{Ad}_{\mathfrak{N}} q^{-1} \quad \text { for } q \in Q, \varphi \in \mathfrak{N}^{*} .
\end{aligned}
$$

Now we recall the definition of an important geometric invariant, the associated variety of an ideal. (More about this invariant, and also some applications to 
representation theory, can be found in Vogan's nicely written paper [V2].) Suppose $I$ is an ideal of the enveloping algebra $U(\mathfrak{N})$ of $\mathfrak{N}$. Then

$$
\operatorname{AV}(I):=\left\{\alpha \in \mathfrak{N}^{*} \mid f(\alpha)=0 \text { for all } f \in \operatorname{gr} I\right\}
$$

is called the associated variety of $I ; \operatorname{AV}(I)$ is a subvariety of the affine variety $\mathfrak{N}^{*}$. (Here; gr $I$ denotes the graded ideal of $I$ in the usual sense. Note that by the Poincaré-Birkhoff-Witt theorem we may regard gr $I$ as an ideal in the symmetric algebra $S(\mathfrak{N})$. Also, the latter may be identified with the algebra of polynomial functions on $\mathfrak{N}^{*}$. This explains why the above definition is correct.)

The following corollary in the case $\mathfrak{Q}=\mathfrak{N}$ becomes a classical result (see, e.g., [Bh, Chap. 2]).

Corollary 5.10. Let $V$ be a $\mathfrak{Q}$-module which is finitely generated as an $\mathfrak{N}$-module. Then the associated variety $\operatorname{AV}(\mathcal{P})$ of an arbitrary foundation prime $\mathcal{P}$ of $V$, when $V$ is regarded as a $U(\mathfrak{N})$-module, is $Q$-stable for the adjoint action of $Q$ on $U(\mathfrak{N})$. Thus, in general, $\operatorname{AV}(\mathcal{P})$ is a finite union of some (coadjoint) $Q$-orbits on $\mathfrak{N}^{*}$.

Proof. This is now clear (by Theorem C and Proposition 5.9).

Remark 5.11. Note that for $\mathfrak{Q}$ and $Q$ as above, the group $\mathcal{G}:=\operatorname{Ad} Q$ is the adjoint group of $\mathfrak{Q}$. Since now $\mathcal{G}$ operates on $\mathfrak{Q}$ by endomorphisms of $\mathfrak{Q}$, in the natural way, we could formulate our results in terms of $\mathcal{G}$-stability and $\mathcal{G}$-orbits instead of $Q$-stability and $Q$-orbits. However, for some concrete computations it is more convenient to work with the group $Q$ (see Fact B.8).

\section{Concluding Remarks}

Throughout this section we fix an algebraically closed field $k$ of characteristic zero.

Suppose $M$ is a finitely generated module over a Noetherian ring $\mathcal{R}$. Then the problem of determining the associated primes Ass $M$ is often very interesting. But even if $\mathcal{R}$ is a pretty "tame" ring (for example, the polynomial algebra in finitely many commuting variables with coefficients from $k$ ), this problem can be very hard. Now, we know that Ass $M$ is a finite set contained in Spec $\mathcal{R}$, and moreover Ass $M \subseteq \mathcal{V}(M)$. The next natural attempt is to further restrict the set of possible candidates for Ass $M$. The main purpose of this section is to describe a variety of situations in which Theorem $\mathrm{C}$ can be of great help in this further restriction of candidates for the associated primes. Also, we will explain some applications relevant for representation theory, and in particular the relation of our work with Casselman's subrepresentation theorem.

Let us now focus on the situation which is the most interesting one for us; that is, suppose we have the setting $G, Q, \mathfrak{g}, \mathfrak{q}, \mathfrak{n}, \mathcal{U}$, etc., as in the first paragraph of the Introduction. Then consider the category

$$
\begin{aligned}
\operatorname{Mod}_{\mathfrak{n}-\text { fin }}(\mathfrak{q}):= & \text { category of } \mathfrak{q} \text {-modules which are finitely generated as } \\
& \mathfrak{n} \text {-modules. }
\end{aligned}
$$

Note. Let us agree on the following. When we speak about the associated primes of a module $V$ from the above category, we always mean that $V$ is considered as a $\mathcal{U}$-module; that is, the associated primes of $V$ are prime ideals of $\mathcal{U}$. Also, we understand that $\mathfrak{q}$ acts on $\mathcal{U}$ via the adjoint action (see (14)). 
Now, Theorem $\mathrm{C}$, when applied to a module $V$ from $\operatorname{Mod}_{\mathfrak{n} \text {-fin }}(\mathfrak{q})$, states that every $\mathcal{P} \in \operatorname{Ass} V$ is q-stable. This crucial fact, which enables us to greatly restrict the set of candidates for Ass $V$, is our main motivation for what follows.

It turns out that it would be useful to consider also some other pairs $(\mathfrak{q}, \mathfrak{n})$ of Lie algebras, where $\mathfrak{q}$ is a parabolic subalgebra of $\mathfrak{g}$ and $\mathfrak{n}$ is a (nilpotent) ideal in $\mathfrak{q}$, and then the corresponding category $\operatorname{Mod}_{\mathfrak{n} \text {-fin }}(\mathfrak{q})$ as defined above. Let us now explain more precisely the general setting in which we are interested. For that purpose, it will be convenient to start with some basics on abstract root systems. Therefore, suppose $R$ is a root system in a finite-dimensional $k$-vector space. Choose a root system basis $B \subseteq R$, and denote $R^{+}:=R \cap \operatorname{span}_{\mathbb{Z}_{+}} B$ and $R^{-}:=-R^{+}$. The next definition is in part a slight variation of the well known one, suitable for our purposes (cf. [B3, Def. 6.1.4]).

Definition 6.1. Suppose $\Gamma$ and $\Omega$ are subsets of $R$ satisfying $\Gamma \subseteq \Omega$. We will say that $\Gamma$ is ideal-closed in $\Omega$ if the following condition holds:

if $\alpha \in \Omega$ and $\beta \in \Gamma$ are such that $\alpha+\beta \in R$, then $\alpha+\beta \in \Gamma$.

In particular, if $\Gamma$ is ideal-closed in $\Gamma$ we say that $\Gamma$ is a closed subset of $R$. For general $\Gamma, \Omega$ as above define the set

$$
\mathrm{I}-\mathrm{Clo}(\Gamma ; \Omega):=\{\Theta \subseteq \Gamma \mid \Theta \text { is ideal-closed in } \Omega\} ;
$$

we understand that $\mathrm{I}-\mathrm{Clo}(\Gamma ; \Omega)$ contains the empty set as well.

A subset $\Pi$ of $R$ is parabolic if it is closed and also satisfies $\Pi \cup(-\Pi)=R$. We will say that $\Pi$ is $R^{+}$-parabolic if it is parabolic and contains $R^{+}$. Denote

$$
\operatorname{Par}\left(R^{+}\right):=\left\{\Pi \subseteq R \mid \Pi \text { is } R^{+} \text {-parabolic }\right\} .
$$

(In the sequel we will consider $\operatorname{I}-\operatorname{Clo}(\Gamma ; \Omega)$ and $\operatorname{Par}\left(R^{+}\right)$as partially ordered sets, ordered by inclusion.)

Now we have the following well known fact (cf. [B3, Prop. 6.1.20]; see also [K] Prop. 5.90]).

Proposition 6.2. For any subset $\Delta \subseteq B$ define the subset

$$
\Pi(\Delta):=R^{+} \cup\left(R \cap \operatorname{span}_{\mathbb{Z}} \Delta\right)
$$

of $R$; in particular, $\Pi(\emptyset)=R^{+}$. Then, the map $\Delta \mapsto \Pi(\Delta)$ is a well defined bijection from the set $\{\Delta \mid \Delta \subseteq B\}$ onto $\operatorname{Par}\left(R^{+}\right)$.

Let $\Pi \in \operatorname{Par}\left(R^{+}\right)$, and then let $\Delta \subseteq B$ be such that $\Pi=\Pi(\Delta)(\Pi(\Delta)$ as in (16)). Define the set

$$
\Gamma(\Delta):=R^{+} \backslash(\Pi(\Delta) \cap(-\Pi(\Delta))) .
$$

Keeping this notation in force, we can establish the next basic lemma (cf. [K] Cor. $5.94])$.

Lemma 6.3. For any $\Delta \subseteq B$, the set $\Gamma(\Delta)$ is ideal-closed in $\Pi(\Delta)$; that is, $\Gamma(\Delta) \in$ $\mathrm{I}-\mathrm{Clo}(\Gamma(\Delta) ; \Pi(\Delta))$.

Note, in particular, that an arbitrary $\Theta \in \mathrm{I}-\mathrm{Clo}\left(R^{+} ; R^{+}\right)$cannot always be obtained as $\Gamma(\Delta)$ for some $\Delta \subseteq B$ (see Example 6.6).

6.4. Suppose $\mathfrak{g}$ is a semisimple Lie algebra over $k$ and let $\mathfrak{h}$ be a Cartan subalgebra in $\mathfrak{g}$. Consider the corresponding root system $R=R(\mathfrak{g}, \mathfrak{h}) ; \mathfrak{g}_{\alpha}$ will denote the root subspace of $\alpha \in R$. Choose a root system basis $B \subseteq R$ which then determines the 
positive (resp. negative) roots $R^{+}$(resp. $R^{-}$). In what follows, for these $R, B$, $R^{+}$and any $\Gamma \subseteq \Omega \subseteq R$, we will freely use the above-introduced $\mathrm{I}-\mathrm{Clo}(\Gamma ; \Omega)$ and $\operatorname{Par}\left(R^{+}\right)$. Also, for $\Delta \subseteq B$, the sets of $\operatorname{roots} \Pi(\Delta)$ and $\Gamma(\Delta)$ will be as defined by (16) and (17), respectively.

Use the above setting and fix some $\Delta \subseteq B$. Then define the parabolic subalgebra (see Proposition 6.2)

$$
\mathfrak{q}(\Delta):=\mathfrak{h} \oplus \bigoplus_{\alpha \in \Pi(\Delta)} \mathfrak{g}_{\alpha}
$$

of $\mathfrak{g}$. Also, define

$$
\mathfrak{u}(\Delta):=\bigoplus_{\alpha \in \Gamma(\Delta)} \mathfrak{g}_{\alpha}
$$

Then $\mathfrak{u}(\Delta)$ is the nilpotent radical of $\mathfrak{q}(\Delta)$ (see Lemma 6.3); note that

$$
\mathfrak{l}(\Delta):=\mathfrak{h} \oplus \bigoplus_{\alpha \in \Pi(\Delta) \cap(-\Pi(\Delta))} \mathfrak{g}_{\alpha}
$$

is the (reductive) Levi factor, and thus we have the Levi decomposition

$$
\mathfrak{q}(\Delta)=\mathfrak{l}(\Delta) \oplus \mathfrak{u}(\Delta) .
$$

Now we are ready to describe the announced general setting; that is, the pairs $(\mathfrak{q}, \mathfrak{n})$ we would like to consider. For that, take an arbitrary $\Theta \in \operatorname{I-Clo}(\Gamma(\Delta) ; \Pi(\Delta))$ and set

$$
\mathfrak{n}=\mathfrak{n}_{\Theta}:=\bigoplus_{\alpha \in \Theta} \mathfrak{g}_{\alpha} \quad \text { and } \quad \mathfrak{q}=\mathfrak{q}_{\Delta}:=\mathfrak{q}(\Delta) .
$$

Then, clearly, $\mathfrak{n}_{\Theta}$ is a nilpotent ideal of $\mathfrak{q}_{\Delta}$ contained in $\mathfrak{u}(\Delta)$, for any such $\Theta$. (Of course, an even more general situation arises by taking for $\mathfrak{q}_{\Delta}$ an arbitrary subalgebra of $\mathfrak{q}(\Delta)$ containing $\mathfrak{n}_{\Theta}$, but the above described pairs $(\mathfrak{q}, \mathfrak{n})$ will suffice for our purposes.)

Remark 6.5. Sometimes it is more convenient to describe the above pairs $(\mathfrak{q}, \mathfrak{n})$ using the description of parabolic subalgebras in $\mathfrak{g}$ starting with an Iwasawa decomposition of $\mathfrak{g}$. More precisely, we proceed as follows (see [D] Sect. 1.13] for details). Let $\mathfrak{g}$ be a semisimple Lie algebra over $k$ possessing an involution $\vartheta$; that is, $(\mathfrak{g}, \vartheta)$ is a semisimple symmetric Lie algebra. Then $\mathfrak{g}=\mathfrak{k} \oplus \mathfrak{p}$, where $\mathfrak{k}:=\{x \in \mathfrak{g} \mid \vartheta x=x\}$ and $\mathfrak{p}:=\{x \in \mathfrak{g} \mid \vartheta x=-x\}$. Also, there exists a Cartan subspace $\mathfrak{a}$ of $\mathfrak{p}$, and so we can define the restricted roots $\Phi=\Phi(\mathfrak{g}, \mathfrak{a})$. For a certain choice of positive roots $\Phi^{+}$, define $\mathfrak{n}:=\bigoplus_{\varphi \in \Phi^{+}} \mathfrak{g}_{\varphi} ; \mathfrak{g}_{\varphi}$ are again the root subspaces. Then we have the Iwasawa decomposition $\mathfrak{g}=\mathfrak{k} \oplus \mathfrak{a} \oplus \mathfrak{n}$. Now proceed as in [K] Sect. 7.7].

The following simple example points at some general phenomena concerning the problem of $\mathfrak{q}$-stability for primes of the enveloping algebra of $\mathfrak{n}$, for $(\mathfrak{q}, \mathfrak{n})$ as described (cf. Appendix B).

Example 6.6. Suppose $\mathfrak{g}:=\mathfrak{s} \mathfrak{l}(3, k)$ and let $\mathfrak{h}$ be a Cartan subalgebra in $\mathfrak{g}$. Then the root system $R=R(\mathfrak{g}, \mathfrak{h})$ is of type $A_{2}$, and so there exists a root system basis $B=\left\{\alpha_{1}, \alpha_{2}\right\}$; then $R^{+}=B \cup\{\mu\}$, where $\mu:=\alpha_{1}+\alpha_{2}$. Further, denote $\Delta_{i}:=\left\{\alpha_{i}\right\}$, for $i=1,2$. Then we have

$$
\mathrm{I}-\mathrm{Clo}\left(R^{+} ; R^{+}\right)=\left\{R^{+}, \Gamma\left(\Delta_{1}\right), \Gamma\left(\Delta_{2}\right),\{\mu\}, \emptyset\right\} .
$$


Consider the Borel subalgebra $\mathfrak{b}:=\mathfrak{q}_{\emptyset}=\mathfrak{h} \oplus \bigoplus_{\alpha \in R^{+}} \mathfrak{g}_{\alpha}$, the parabolic subalgebra $\mathfrak{q}:=\mathfrak{q}_{\Delta_{1}}=\mathfrak{g}_{-\alpha_{1}} \oplus \mathfrak{b}$ and the nilpotent subalgebra $\mathfrak{n}:=\mathfrak{n}_{\Gamma\left(\Delta_{1}\right)}$ of $\mathfrak{g}$ (see (18)); note that $\mathfrak{n}$ is an ideal in $\mathfrak{q}$ (and so, also in $\mathfrak{b}$ ). Now we have the following:

Claim. The questions of $\mathfrak{b}$-stability and $\mathfrak{q}$-stability for primes of the enveloping algebra $U(\mathfrak{n})$ of $\mathfrak{n}$ are not equivalent.

[Let $\mathcal{P}$ denote the (prime) ideal $U(\mathfrak{n}) \mathfrak{g}_{\mu}$ of $U(\mathfrak{n})$. It is easy to check that the prime $\mathcal{P}$ is $\mathfrak{b}$-stable but not $\mathfrak{q}$-stable.]

For further needs we also give the next easy lemma.

Lemma 6.7. Let $\mathfrak{g}, \mathfrak{h}, R, \mathfrak{g}_{\alpha}$ for $\alpha \in R, B$ and $R^{+}$be as in 6.4. Choose some $\Delta \subseteq B$ and $\Theta \in \mathrm{I}-\operatorname{Clo}(\Gamma(\Delta) ; \Pi(\Delta))$, and let $\mathfrak{n}=\mathfrak{n}_{\Theta}$ and $\mathfrak{q}=\mathfrak{q}_{\Delta}$ be as in (18). Denote by $\mathcal{U}$ the enveloping algebra of $\mathfrak{n}$ (and consider the adjoint $\mathfrak{q}$-action on it, as we agreed before).

(i) Suppose that $\mathfrak{r}$ is an ideal of $\mathfrak{q}$, contained in $\mathfrak{n}$ (e.g., $\mathfrak{r}:=\bigoplus_{\alpha \in \Upsilon} \mathfrak{g}_{\alpha}$ for some $\Upsilon \in \operatorname{I-Clo}(\Gamma(\Delta) ; \Pi(\Delta))$ contained in $\Theta)$. Then $\mathcal{U} \mathfrak{r}$ is a $\mathfrak{q}$-stable (two-sided) ideal of $\mathcal{U}$.

(ii) The left ideal $\mathcal{U} X_{\alpha}$ is $\mathfrak{h}$-stable, for any $\alpha \in \Theta$; here $X_{\alpha}$ is a vector from $\mathfrak{g}_{\alpha}$. (Caution. In general, $\mathcal{U} X_{\alpha}$ is not $\mathfrak{q}$-stable!)

Proof. (i) By [D, 2.2.14 Prop.] we know that $\mathcal{U} \mathfrak{r}$ is a two-sided ideal of $\mathcal{U}$. Further, for arbitrary $q \in \mathfrak{q}, u \in \mathcal{U}$ and $r \in \mathfrak{r}$ we have

$$
[q, u r]=[q, u] r+u[q, r] .
$$

Since obviously $[q, u] \in \mathcal{U}$, the claim follows.

(ii) Put $h \in \mathfrak{h}$ and $X_{\alpha}$ in place of $q$ and $r$, respectively, in (20).

Suppose $\mathfrak{Q}$ is a Lie algebra over $k$. Let $\mathfrak{Q}^{*}$ be its dual, and $\mathcal{G}$ its algebraic adjoint group. Then $\mathcal{G}$ operates on $\mathfrak{Q}^{*}$ in the obvious way. Now, if $\mathfrak{Q}$ is moreover solvable, then the Dixmier map (see, e.g., [D, Sect. 6])

$$
\operatorname{Dix}: \mathfrak{Q}^{*} / \mathcal{G} \longrightarrow \operatorname{Prim} U(\mathfrak{Q})
$$

is a (homeomorphic) bijection from the set of all the coadjoint $\mathcal{G}$-orbits on $\mathfrak{Q}^{*}$ onto the set of all the primitive ideals of the enveloping algebra of $\mathfrak{Q}$. This is a deep result relating certain fairly easy computable geometric objects (orbits), and certain purely algebraic objects (primitive ideals), understanding which is, in general, more complicated.

Suppose now that $\mathfrak{q}$ is an algebraic Lie algebra in $\mathfrak{g l}(n, k), \mathfrak{n}$ is an ideal of $\mathfrak{q}$, and $Q$ is a connected closed subgroup of $G L(n, k)$ so that $\mathfrak{q}$ is its Lie algebra. (Then Ad $Q$ is the adjoint group of $\mathfrak{q}$; see Remark 5.11.) Define

$$
\operatorname{Spec}^{Q} U(\mathfrak{n}):=\text { set of } Q \text {-stable prime ideals of } U(\mathfrak{n}) .
$$

The following corollary provides the first basic information on the desired restriction of the set of possible candidates for Ass $V$; it is an immediate consequence of Theorem $\mathrm{C}$ and Proposition 5.9 .

Corollary 6.8. Suppose $\mathfrak{q}, \mathfrak{n}$ and $Q$ are as above. Let $V$ be an arbitrary module from the category $\operatorname{Mod}_{\mathfrak{n} \text {-fin }}(\mathfrak{q})$. Then

$$
\text { Ass } V \subseteq \operatorname{Spec}^{Q} U(\mathfrak{n}) .
$$

Moreover, Fnd $V \subseteq \operatorname{Spec}^{Q} U(\mathfrak{n})$. 
Assume now the setting as in 6.4 and let $(\mathfrak{q}, \mathfrak{n})$ be a pair as given by (18). Our next aim here is to point at the fact which indicates that there exists a certain (bijective!?) relation between the set $\mathfrak{n}^{*} / Q$ of coadjoint $Q$-orbits on $\mathfrak{n}^{*}$ and the set $\operatorname{Spec}^{Q} U(\mathfrak{n})$. (This should be a generalization of the Dixmier map, by means of which the more complicated problem of understanding $\operatorname{Spec}^{Q} U(\mathfrak{n})$ could be "transferred" to the easier problem of computing the coadjoint orbits.) Besides, we will see that it is quite reasonable to expect that the latter set is an appropriate choice for the mentioned candidates via which we would like to study Ass $V$. Namely, $\operatorname{Spec}^{Q} U(\mathfrak{n})$ is not too big; moreover, it seems that this set will often be finite. Before we give the announced fact we would like to say how it appears to us to be somewhat surprising. A good explanation of what is going on in the general situation would be very valuable.

Fact 6.9. Let $\mathfrak{g}, \mathfrak{h}, R, B$ and $R^{+}$be as in 6.4 $\Delta \subseteq B$ and $\Theta \in \operatorname{I-Clo}(\Gamma(\Delta) ; \Pi(\Delta))$. Let $\mathfrak{n}=\mathfrak{n}_{\Theta}$ and $\mathfrak{q}=\mathfrak{q}_{\Delta}$ be as in (18). Also, let $Q$ be a connected algebraic group so that $\mathfrak{q}$ is its Lie algebra, as before. Now, consider the following particular choice: Let $\mathfrak{g}$ be either $\mathfrak{s l}(2, k)$ or $\mathfrak{s l}(3, k), \Delta:=\emptyset$ and $\Theta:=R^{+}$. Then there are "natural" bijective maps $\beta$ and $\delta$,

$$
\mathrm{I}-\mathrm{Clo}(\Gamma(\Delta) ; \Pi(\Delta)) \stackrel{\beta}{\longrightarrow} \mathfrak{n}^{*} / Q \stackrel{\delta}{\longrightarrow} \operatorname{Spec}^{Q} U(\mathfrak{n}) .
$$

(Note that here $\Gamma(\Delta)=\Pi(\Delta)=R^{+}$, and $\mathfrak{q}$ is the minimal parabolic subalgebra with the Levi decomposition $\mathfrak{h} \oplus \mathfrak{n}$.)

[For $\mathfrak{g}=\mathfrak{s l}(2, k)$, combine the Claim in the proof of Lemma A.4 with Fact A.6 For $\mathfrak{g}=\mathfrak{s l}(3, k)$ combine (19), Theorem B.3 and Fact B.8.]

Remark 6.10. (1) For general $(\mathfrak{q}, \mathfrak{n})$ as in (18) and the corresponding group $Q$, the zero-orbit will be the only closed $Q$-orbit on $\mathfrak{n}^{*}$. Also, the augmentation ideal $\mathcal{A}$ of $U(\mathfrak{n})$ is $Q$-stable (equiv. q-stable; the latter by Lemma 6.7(i)); that is, $\mathcal{A} \in$ $\operatorname{Spec}^{Q} U(\mathfrak{n})$. It will be the only $Q$-stable primitive ideal of $U(\mathfrak{n})$; consequently, for a finite-dimensional $\mathfrak{q}$-module we will have $\operatorname{Ass} V=\operatorname{Supp}(V)=\{\mathcal{A}\}$ (see Proposition 4.1).

(2) Let $\mathfrak{Q}$ be any Lie algebra and $\mathcal{G}$ its algebraic adjoint group. It is a classical result that every ideal of the enveloping algebra $U(\mathfrak{Q})$ is $\mathcal{G}$-stable; this is a consequence of the obvious fact that every such ideal is stable under all the inner

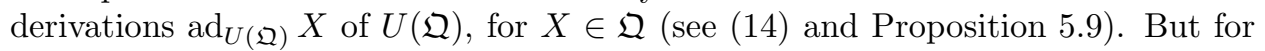
$(\mathfrak{q}, \mathfrak{n})$ and $Q$ as in (1), the question of $Q$-stability for the primes of $U(\mathfrak{n})$ is more subtle. (The latter is equivalent, again by Proposition 5.9, to the question of stability for such primes under all the outer derivations of $U(\mathfrak{n})$ having the form $\operatorname{ad}_{U(\mathfrak{n})} X$, $X \in \mathfrak{q} \backslash \mathfrak{n}$.)

Let us now explain the relation of our work with Casselman's theorem given in the first paragraph of the Introduction; for that purpose suppose again that we have the setting given there. Let $V$ be an admissible finitely generated $(\mathfrak{g}, K)$ module. Then Casselman's theorem says that $\mathcal{A} V \neq V$, which is, by Theorem 4.3, further equivalent to $\mathcal{A} \in \operatorname{Supp}(V)$ (see the Note at the beginning of this section). Also, recall that by the well known Osborne's lemma [CO, 2.3 Thm.] we know that $V$ is a finitely generated $\mathcal{U}$-module; therefore, in particular, $V$ is from the category $\operatorname{Mod}_{\mathfrak{n} \text {-fin }}(\mathfrak{q})$. Motivated by this, we formulate the following problem, whose understanding would be very interesting, both for representation theory and for "pure" noncommutative algebra. For that, suppose we have the setting as in 
6.4, and let $(\mathfrak{q}, \mathfrak{n})$ be as in (18); the case when $\mathfrak{q}$ is a minimal parabolic subalgebra of $\mathfrak{g}$ is of particular interest. Denote, as always, the enveloping algebra of $\mathfrak{n}$ by $\mathcal{U}$, and the augmentation ideal of $\mathcal{U}$ by $\mathcal{A}$.

Problem. Find some subcategory $\mathcal{C}$ of the category $\operatorname{Mod}_{\mathfrak{n}-\mathrm{fin}}(\mathfrak{q})$ such that for any module $V$ from $\mathcal{C}$ we have $\mathcal{A} \in \operatorname{Supp}(V), V$ considered as a $\mathcal{U}$-module; and more generally, such that we can describe the set of all the maximal primes within $\operatorname{Supp}(V)$ and/or characterize it via some accessible "geometric condition" (see Remark 6.12 (3) below). In particular, assuming the standard setting of the Introduction (then $k=\mathbb{C}$ ), find some $\mathcal{C}$ containing the restrictions of admissible finitely generated $(\mathfrak{g}, K)$-modules to $\mathfrak{n}$.

Casselman and Osborne suggested the existence of such a category $\mathcal{C}$ as above for which one should be able to adapt their proof of Casselman's theorem for $\mathfrak{n}$ abelian to the case of nonabelian $\mathfrak{n}$ (cf. [CO, p.198]); now, $\mathfrak{q}$ is a minimal parabolic subalgebra of $\mathfrak{g}$. (Taking into account Remark B.7(1), we see that the category $\mathcal{C}$ we are searching for must be strictly smaller than $\operatorname{Mod}_{\mathfrak{n} \text {-fin }}(\mathfrak{q})$; cf. [SW].) Here we propose such one category $\mathcal{C}$. (Note that for $G, K$, $\mathfrak{g}$, etc., using just Casselman's theorem, it follows that $\mathcal{C}$ obviously contains the restrictions of admissible finitely generated $(\mathfrak{g}, K)$-modules to $\mathfrak{n}$; of course, it would be nice to have an independent argument for the latter fact.) Namely, define $\mathcal{C}=\mathcal{C}(\mathfrak{q}, \mathfrak{n})$ to be the subcategory of $\operatorname{Mod}_{\mathfrak{n} \text {-fin }}(\mathfrak{q})$ consisting of modules $V$ satisfying the following condition:

$(\diamond)$ There exists a nontrivial q-subquotient $Y(V)$ of $V$ such that $Y(V)$ has a simple $\mathfrak{n}$-submodule $E(V)$.

Claim 6.11. If $V$ is from $\mathcal{C}$, then $\mathcal{A} \in \operatorname{Supp}(V)$.

[Sketch of proof. Let $Y(V)$ and $E(V)$ be as in $(\diamond)$, and denote $\mathcal{P}:=\operatorname{ann}_{\mathcal{U}}(E(V))$. Then, by Theorem $\mathrm{C}$ and Corollary 4.2 we know that $\mathcal{P}$ is a $\mathfrak{q}$-stable associated prime of $Y(V)$. Further, by Corollary 5.10 one should conclude that the associated variety $\operatorname{AV}(\mathcal{P})$ equals the zero-orbit of $Q$ on the dual of $\mathfrak{n}$, where $Q$ is as in the corollary; thus $\mathcal{P}=\mathcal{A}$ (cf. Remark 6.10(1)). Finally, by Corollary 4.5] and Theorem 4.3, we have $\mathcal{A} \in \operatorname{Supp}(V)$.]

We will conclude this section with the following conjecture and some related comments. (Proposition 4.1 and Corollary B.6 provide the first moral support to believe in it. But let us say that we think this conjecture in full generality could be very hard.)

Conjecture. Suppose we have the setting as in the first paragraph of the Introduction, and let $V$ be an admissible finitely generated $(\mathfrak{g}, K)$-module. Then

$$
\text { Ass } V \subseteq \operatorname{Supp}(V) \text {; }
$$

or, equivalently (see Corollary [2.5, Lemma 2.8 and Theorem [2.10(ii)),

$$
\mathcal{V}(V)=\operatorname{Supp}(V) \text {. }
$$

Remark 6.12. (1) The above conjecture aims to provide a sharp generalization of Casselman's theorem stated in the Introduction. Namely, the understanding of the $\operatorname{support} \operatorname{Supp}(V)$ should give all the maximal ideals $\mathcal{M}$ of $\mathcal{U}$ such that $V / \mathcal{M} V \neq 0$ (cf. Theorem A and (2) below). Let us also say that, when considering the latter non-vanishing condition on $V$, the augmentation ideal $\mathcal{A}$ is in fact the simplest one of $\mathcal{M}$ with which we have to deal. 
(2) Suppose $V$ is an infinite-dimensional irreducible admissible $(\mathfrak{g}, K)$-module. Using Corollaries 4.9 and 6.8 and Remark 6.10(1), we deduce that Ass $V$ does not contain any primitive (equiv. maximal) ideal of $\mathcal{U}$. Now, assuming the above conjecture is true, we would have that there are (infinitely) many maximal ideals $\mathcal{M}$ as in (1); of course, one of these is the augmentation ideal $\mathcal{A}$ itself.

(3) Suppose $V$ is a finitely generated $\mathcal{U}$-module. Then for any (maximal) prime $I \in \mathcal{V}(V)$ we may compute the associated variety $\operatorname{AV}(I)$, as explained in Sect. 5 The latter leads us to consider the set $\mathcal{V}(V)$ as a geometric object. In other words, using this relation between the primes of $\mathcal{V}(V)$ and their associated varieties, one can in principle obtain a fairly satisfactorily understanding of $\mathcal{V}(V)$. In particular, we may hope to deal successfully with the problem of whether $\mathcal{V}(V)$ contains the augmentation ideal $\mathcal{A}$ of $\mathcal{U}$. However, in general, the condition $\mathcal{A} \in \mathcal{V}(V)$ is not so closely related to the non-vanishing condition $V / \mathcal{A} V \neq 0$ of Casselman's theorem (cf. Theorem 4.3). But, as we noted before, the latter is equivalent to the condition $\mathcal{A} \in \operatorname{Supp}(V)$. That is that $\mathcal{A}$ belongs to a certain set of primes which may be understood as a purely algebraic object, defined via the localization, computing which could be in principle pretty difficult. This further explains why we would like to know for which $\mathcal{U}$-modules $V$ the (geometric object) $\mathcal{V}(V)$ is equal to the (algebraic object) $\operatorname{Supp}(V)$. (Supposing $\mathfrak{n}$ abelian, the equality $\mathcal{V}(V)=\operatorname{Supp}(V)$ for every $V$ was in fact the crucial reason which, in combination with Theorem $\mathrm{C}$, enabled Casselman and Osborne [CO] to conclude that $\mathcal{A} \in \mathcal{V}(V)$ (equiv. $V / \mathcal{A} V \neq$ $0)$, for every admissible finitely generated $(\mathfrak{g}, K)$-module.)

Let us denote by min-Ass $V$ the set of minimal elements of Ass $V$. Obviously, the above conjecture is further equivalent to the inclusion

$$
\min -\operatorname{Ass} V \subseteq \operatorname{Supp}(V) \text {. }
$$

The next proposition shows that the latter is in fact equivalent to the problem of checking whether the annihilators of certain elements from some foundation layers of $V$ are moreover two-sided ideals. (We freely use below the notation introduced in Definition 1.2; ;f. also the proof of [Š2, Thm. 2.5].)

Proposition 6.13. Let $\mathcal{U}$ be the enveloping algebra of a nilpotent Lie algebra. Let $V$ be a finitely generated $\mathcal{U}$-module and $\mathcal{P}$ a minimal associated prime of $V$. Then $\mathcal{P} \in \operatorname{Supp}(V)$ if and only if there exists $1 \leq p \leq \mathrm{ff}-\mathrm{l}(V)$ and a nonzero $v \in \mathcal{L}^{p}(V)$ such that

(1) $\mathcal{P}=\mathfrak{A}_{v}$, and

(2) $\operatorname{ann}(v)$ is a two-sided ideal of $\mathcal{U}$.

Proof. Suppose that $\mathcal{P} \in \operatorname{Supp}(V)$ and at the same time there are no $p$ and $v$ satisfying (1) and (2). Then take an arbitrary $x \in V$ and assume that $x \in \mathcal{F}_{i_{0}}(V)$, but $x \notin \mathcal{F}_{i_{0}-1}(V)$, for some $i_{0}$. Write $\mathcal{L}^{i}(V)=\bigoplus_{j=1}^{\kappa_{i}} F_{j}^{i}$ for every $1 \leq i \leq i_{0}$ and certain $\kappa_{i} \in \mathbb{N}$, and denote $\mathcal{P}_{j}^{i}:=\operatorname{ann}\left(F_{j}^{i}\right)$ (cf. Proposition 1.1(ii)); thus $\operatorname{Fnd}_{i} V=\left\{\mathcal{P}_{1}^{i}, \ldots, \mathcal{P}_{\kappa_{i}}^{i}\right\}$. Accordingly, write

$$
\mathcal{L}^{i_{0}}(V) \ni x+\mathcal{F}_{i_{0}-1}(V)=\sum_{j=1}^{\kappa_{i_{0}}} \gamma_{j}^{i_{0}}, \quad \gamma_{j}^{i_{0}} \in F_{j}^{i_{0}} .
$$

Now, if $\mathcal{P} \neq \mathcal{P}_{j}^{i_{0}}$ (resp. $\mathcal{P}=\mathcal{P}_{j}^{i_{0}}$ ), let $\sigma_{j}^{i_{0}}$ be an arbitrary element from $\mathcal{P}_{j}^{i_{0}}$ (resp. $\left.\operatorname{ann}\left(\gamma_{j}^{i_{0}}\right)\right)$ not belonging to $\mathcal{P}$. Set $\sigma^{i_{0}}:=\prod_{j=1}^{\kappa_{i_{0}}} \sigma_{j}^{i_{0}}$ and $x_{i_{0}-1}:=\sigma^{i_{0}} x$, and then 
note that $x_{i_{0}-1} \in \mathcal{L}^{i_{0}-1}(V)$. Proceeding inductively, in finitely many steps we can find some $\sigma \in \mathcal{U} \backslash \mathcal{P}$ such that $\sigma x=0$. Hence, $V_{\mathcal{P}}=0$; a contradiction.

For the opposite implication, first note that Lemma 6.14 below gives $\mathcal{P} \in$ $\operatorname{Supp}\left(\mathcal{L}^{p}(V)\right)$. Hence, by means of Proposition [2.3, we deduce that $\mathcal{P} \in \operatorname{Supp}(V)$.

Lemma 6.14. Let $\mathcal{R}$ be a left Noetherian ring and $M$ a finitely generated $\mathcal{R}$ module. For an arbitrary nonzero $m \in M$ denote by $L_{m}$ the left ideal $\operatorname{ann}(m)$. If $L_{m}$ is a two-sided ideal of $\mathcal{R}$, then $L_{m}=\mathfrak{A}_{m}$. If $L_{m}$ is moreover a prime ideal, that is $L_{m} \in \operatorname{Annspec} M$, then $L_{m} \in \operatorname{Supp}(M)$.

Proof. The first claim is clear; for the second one, use the fact that $m / 1$ is now a nonzero element from the localization of $M$ at $L_{m}$ (see Proposition 2.9).

\section{Appendix A. Some computations for $\mathfrak{s l}(2)$-Modules}

The following notation will be fixed throughout this appendix (cf. the first paragraph in the Introduction). Let $G:=S L(2, \mathbb{R})$, and

$$
K:=S O(2, \mathbb{R})=\left\{\left(\begin{array}{cc}
\cos \theta & -\sin \theta \\
\sin \theta & \cos \theta
\end{array}\right) \mid \theta \in \mathbb{R}\right\},
$$

a maximal compact subgroup of $G$. Take a minimal parabolic subgroup

$$
Q:=\left\{\left(\begin{array}{cc}
x & y \\
0 & x^{-1}
\end{array}\right) \mid x \in \mathbb{R}^{\times}, y \in \mathbb{R}\right\}
$$

of $G$. Then $Q=M A N$ is the Langlands decomposition, where

$$
M:=\left\{\left(\begin{array}{ll}
1 & 0 \\
0 & 1
\end{array}\right),\left(\begin{array}{cc}
-1 & 0 \\
0 & -1
\end{array}\right)\right\}, \quad A:=\left\{\left(\begin{array}{cc}
t & 0 \\
0 & t^{-1}
\end{array}\right) \mid t>0\right\}
$$

and

$$
N:=\left\{\left(\begin{array}{ll}
1 & r \\
0 & 1
\end{array}\right) \mid r \in \mathbb{R}\right\}
$$

Also, denote

$$
k:=\left(\begin{array}{cc}
0 & -1 \\
1 & 0
\end{array}\right), \quad h:=\left(\begin{array}{cc}
1 & 0 \\
0 & -1
\end{array}\right), \quad e:=\left(\begin{array}{ll}
0 & 1 \\
0 & 0
\end{array}\right) .
$$

Then $\mathfrak{g}_{0}=\mathfrak{s l}(2, \mathbb{R}), \mathfrak{k}_{0}=\mathbb{R} k, \mathfrak{a}_{0}=\mathbb{R} h, \mathfrak{n}_{0}=\mathbb{R} e$ and $\mathfrak{q}_{0}=\mathbb{R} h \oplus \mathbb{R} e$ are the Lie algebras of $G, K, A, N$ and $Q$, respectively. Let $\mathfrak{g}, \mathfrak{k}, \mathfrak{a}, \mathfrak{n}$ and $\mathfrak{q}$ be the corresponding complexifications. Further, define the following elements of $\mathfrak{g}$ :

$$
\bar{k}:=\imath k, \quad n^{+}:=\frac{1}{2}\left(\begin{array}{cc}
1 & \imath \\
\imath & -1
\end{array}\right), \quad n^{-}:=\frac{1}{2}\left(\begin{array}{cc}
1 & -\imath \\
-\imath & -1
\end{array}\right)
$$

( $\iota$ denotes the imaginary unit). Also, let $\mathcal{U}$ be the universal enveloping algebra of $\mathfrak{n}$; note that $\mathcal{U}=\mathbb{C}[e]$, the polynomial algebra in the variable $e$. By $\Omega$ we denote the Casimir element $4 n^{+} n^{-}+\bar{k}^{2}-2 \bar{k}$ of $U(\mathfrak{g})$, and as usual $\mathcal{A}$ is the augmentation ideal of $\mathcal{U}$.

Now, by the general theory it follows that any admissible $(\mathfrak{g}, K)$-module $V$ which is moreover quasisimple (that is, the Casimir element $\Omega$ acts on $V$ as a multiple of the identity $1_{V}$ ) is a direct sum of standard $\mathfrak{g}$-modules (see [HT] Chap. II and, in particular, Thm. 1.3.1]). Hence, any such $V$ is a direct sum of indecomposable, 
quasisimple and $\bar{k}$-multiplicity-free $\mathfrak{g}$-modules. For further needs we recall the following result about the structure of the latter modules (cf. [HT] Thm. II.1.1.13 and Prop. II.1.1.4]).

Theorem A.1. Let $X$ be any indecomposable, $\bar{k}$-multiplicity-free and quasisimple $\mathfrak{g}$-module such that the Casimir element $\Omega$ acts on $X$ by a scalar $\mu \in \mathbb{C}$. Then there exist a scalar $\lambda \in \mathbb{C}$, an interval $I$ in $\mathbb{Z}$ and a $\bar{k}$-eigenbasis $\left\{v_{i} \mid i \in I\right\}$ for $X$ such that the following relations hold:

$$
\begin{aligned}
\bar{k} v_{i} & =(\lambda+2 i) v_{i}, \\
n^{+} v_{i} & =\pi_{i} v_{i+1}, \\
n^{-} v_{i} & =\eta_{i} v_{i-1}, \quad \text { for } i \in I .
\end{aligned}
$$

Here $\pi_{i}$ and $\eta_{i}$ are certain scalars which can be written explicitly as functions in $\mu$, $\lambda$ and $i$, and such that $\pi_{i} \neq 0$ (resp. $\left.\eta_{i} \neq 0\right)$ when $i+1 \in I($ resp. $i-1 \in I)$; in particular, when $i+1 \notin I$ (resp. $i-1 \notin I$ ) we read the above relations as $n^{+} v_{i}=0$ (resp. $\left.n^{-} v_{i}=0\right)$.

Proposition A.2. Let $V$ be a $\mathfrak{g}$-module. Suppose that $V$ has an infinite-dimensional submodule $X$ which is indecomposable, $\bar{k}$-multiplicity-free and quasisimple as $a \mathfrak{g}$-module. Then there exists $w \in X$ such that $s w \neq 0$ for every nonzero $s \in \mathcal{U}$.

Proof. (We freely use the notation from Theorem A.1.)

We claim that $w$ can be chosen, for example, as $v_{i_{0}}$ for some $i_{0} \in I$. To see this, first note that every nonzero $s \in \mathcal{U}$ can be written as

$$
s=a_{0}+a_{1} e+\cdots+a_{m} e^{m} \quad \text { for } a_{i} \in \mathbb{C}, a_{m} \neq 0 .
$$

Now, since $e=(\imath / 2)\left(\bar{k}+n^{-}-n^{+}\right)$, for arbitrary $j \in I$ we have

$$
s v_{j}=\sum_{k=j-m}^{j+m} \gamma_{k} v_{k} \quad \text { for } \gamma_{k} \in \mathbb{C},
$$

where obviously for the (lowest and highest) leading coefficients we have

$$
\gamma_{j-m}=a_{m}\left(\frac{\imath}{2}\right)^{m}\left(\prod_{l=0}^{m-1} \eta_{j-l}\right) \quad \text { and } \quad \gamma_{j+m}=a_{m}\left(\frac{-\imath}{2}\right)^{m}\left(\prod_{l=0}^{m-1} \pi_{j+l}\right) .
$$

To finish the proof it remains to observe that we can find $i_{0} \in I$ such that either $\gamma_{i_{0}-m} \neq 0$ for all $m \in \mathbb{N}$, or $\gamma_{i_{0}+m} \neq 0$ for all $m \in \mathbb{N}$; or equivalently said, that either $\eta_{i_{0}-l} \neq 0$ for every $l \in \mathbb{N}$, or $\pi_{i_{0}+l} \neq 0$ for every $l \in \mathbb{N}$. Namely, if $X$ has a finite-dimensional submodule $F$, then any $i_{0}$ for which $v_{i_{0}} \notin F$ will certainly do. (Using the diagram-notation as in [HT, Thm. II.1.1.13], the possible types for $X$ are $(\circ[\circ] \circ),(\circ[\circ])$ and $([\circ] \circ)$; then the type of $F$ is $([\circ])$.) For all the other types of $X, i_{0} \in I$ can be chosen arbitrarily.

The following corollary is now an immediate consequence of the above proposition (see Corollary 2.5 and Theorem 2.10(i)).

Corollary A.3. Let $V$ be as in Proposition A.2, and let $w \in X$ be chosen as there. Then for $V$, considered as a $\mathcal{U}$-module, we have $\mathfrak{A}_{w}=(0) \in$ Ass $V$, and hence

$$
\mathcal{V}(V)=\operatorname{Supp}(V)=\operatorname{Spec} \mathcal{U}
$$

as a consequence we have $\mathcal{P} V \neq V$ for all primes $\mathcal{P}$ of $\mathcal{U}$. 
Before we formulate the main result of this appendix (Theorem A.5), let us establish the next simple auxiliary lemma (cf. Remark 6.10(1)).

Lemma A.4. Let $V$ be a finite-dimensional $\mathfrak{q}$-module. Then the augmentation ideal $\mathcal{A}$ is the only associated prime of $V$, where $V$ is considered as a $\mathcal{U}$-module.

Proof. Denote by $\mathcal{P}_{\gamma}$ the maximal ideal of $\mathcal{U}=\mathbb{C}[e]$ generated by $e-\gamma, \gamma \in \mathbb{C}$; then Spec $\mathcal{U}=\{(0)\} \cup\left\{\mathcal{P}_{\gamma} \mid \gamma \in \mathbb{C}\right\}$. Straightforward verification proves the following claim (cf. Theorem B.3 and Fig. 1).

Claim. The primes $\mathcal{P}_{0}=\mathcal{A}$ and (0) are the only ones which are $\mathfrak{q}$-stable.

Now let $\mathcal{P}$ be an associated prime of $V$. Then, by Theorem $\mathrm{C}$ and the above Claim, we know that either $\mathcal{P}=\mathcal{A}$ or $\mathcal{P}=(0)$. Since $\mathcal{P}$ must be a maximal ideal (by Proposition 4.1), we conclude that $\mathcal{P}=\mathcal{A}$.

Theorem A.5. Let $V$ be an admissible finitely generated $(\mathfrak{g}, K)$-module. Then for $V$, considered as a $\mathcal{U}$-module, we have

$$
\begin{aligned}
\operatorname{Ass} V=\operatorname{Supp}(V)=\{\mathcal{A}\} & \text { if } \operatorname{dim} V<\infty, \\
(0) \in \operatorname{Ass} V \quad \text { and } \quad \operatorname{Supp}(V)=\operatorname{Spec} \mathcal{U} & \text { if } \operatorname{dim} V=\infty .
\end{aligned}
$$

In particular, in the case $\operatorname{dim} V=\infty$ we have $\mathcal{P} V \neq V$ for all the primes $\mathcal{P}$ of $\mathcal{U}$.

Proof. The case $\operatorname{dim} V<\infty$ follows by Proposition 4.1 and LemmaA.4. If $\operatorname{dim} V=$ $\infty$, then there exists an infinite-dimensional submodule $X$ as in Proposition A.2 here we use the condition that $V$ is finitely generated. Now we apply Corollary A.3.

Let

$$
Q_{\mathbb{C}}:=\left\{\left(\begin{array}{cc}
w & z \\
0 & w^{-1}
\end{array}\right) \mid w \in \mathbb{C}^{\times}, z \in \mathbb{C}\right\}, \quad L_{\mathbb{C}}:=\left\{\left(\begin{array}{cc}
\tau & 0 \\
0 & \tau^{-1}
\end{array}\right) \mid \tau \in \mathbb{C}^{\times}\right\}
$$

and

$$
N_{\mathbb{C}}:=\left\{\left(\begin{array}{ll}
1 & s \\
0 & 1
\end{array}\right) \mid s \in \mathbb{C}\right\}
$$

be the complexifications of the groups $Q, L:=M A$ and $N$, respectively. Consider the adjoint representation of $Q_{\mathbb{C}}$ on $\mathfrak{n}$ (see (12) and (13)), and the corresponding coadjoint representation; that is, the coadjoint action of $Q_{\mathbb{C}}$ on the dual $\mathfrak{n}^{*}$ of $\mathfrak{n}$ (see (15)). Since $n . \varphi=\varphi$, for any $n \in N_{\mathbb{C}}$ and $\varphi \in \mathfrak{n}^{*}$, it is clear that $\mathfrak{n}^{*} / N_{\mathbb{C}} \equiv \mathfrak{n}^{*}$; that

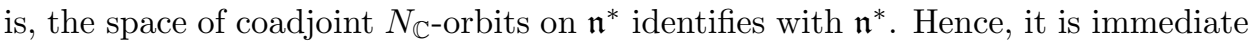

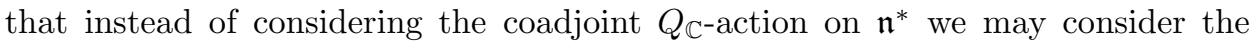
coadjoint $L_{\mathbb{C}^{-}}$action. We will finish this appendix with the following fact; its simple verification is left to the reader.

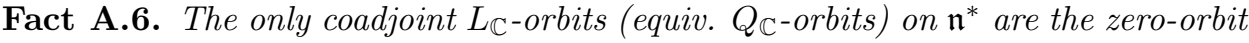
$\{0\}$ and the complement $\mathfrak{n}^{*} \backslash\{0\}$ of the zero-orbit.

Appendix B. Spec $U(\mathfrak{n})$ And $\mathfrak{q}$-STABIlity; Case $\mathfrak{g}=\mathfrak{s} l(3)$

If not otherwise said, the following notation will be fixed in this appendix. Fix an algebraically closed field $k$ of characteristic zero, and denote $k^{\times}:=k \backslash\{0\}$ and $\mathfrak{g}:=\mathfrak{s l}(3, k)$. Let $E_{i j}$, for $1 \leq i, j \leq 3$, denote the standard $3 \times 3$ matrix having 1 in the place $(i, j)$ and 0 elsewhere. Set $x:=E_{12}, y:=E_{23}$ and $z:=E_{13}$. Then $\mathfrak{n}:=k x \oplus k y \oplus k z$ is the three-dimensional (nilpotent, nonabelian) Heisenberg 
Lie algebra over $k$ (cf. Example 2.11). Also, let $\mathfrak{l}$ be the Cartan subalgebra of $\mathfrak{g}$ consisting of all diagonal $3 \times 3$ matrices of trace zero. Denote by $\mathfrak{q}$ the minimal parabolic subalgebra $\mathfrak{l} \oplus \mathfrak{n}$ of $\mathfrak{g}$. As before, we write $\mathcal{U}$ for the universal enveloping algebra of $\mathfrak{n}$ and $\mathcal{A}$ for its augmentation ideal.

The main purpose of this appendix is to show how Theorem C considerably restricts the set of possible candidates for the annihilator (equiv. associated) primes of modules from $\operatorname{Mod}_{\mathfrak{n} \text {-fin }}(\mathfrak{q})$ (see Theorem B.3); $\operatorname{Mod}_{\mathfrak{n} \text {-fin }}(\mathfrak{q})$ is the same category as defined in Sect. 6. We will do this using a rather simple explicit description of the prime spectrum Spec $\mathcal{U}$. For convenience of the reader we explain this description in detail (cf. [BGR, 5.7 Beispiel]).

First, define the closed subset

$$
V(z):=\mathcal{V}(\mathcal{U} z)=\{\mathcal{P} \in \operatorname{Spec} \mathcal{U} \mid \mathcal{P} \supseteq \mathcal{U} z\}
$$

of Spec $\mathcal{U}$, determined by $z$; and then define its complement in $\operatorname{Spec} \mathcal{U}$, that is, the "distinguished open set" determined by $z$,

$$
D(z):=\operatorname{Spec} \mathcal{U} \backslash V(z) .
$$

Now, by Proposition 3.4, the map

$$
\phi_{1}: D(z) \longrightarrow \operatorname{Spec}_{z}, \quad \phi_{1}(\mathcal{P}):=\mathcal{P}_{z},
$$

is a homeomorphism; here by $\mathcal{U}_{z}$ and $\mathcal{P}_{z}$ we denote the localization at $z$, that is, the localization with respect to the denominator set $\left\{1, z, z^{2}, \ldots\right\}$, of the algebra $\mathcal{U}$ and the ideal $\mathcal{P}$, respectively. Further, it can be shown that the localization $\mathcal{U}_{z}$ is isomorphic as an algebra to $k[z]_{z} \otimes \mathbb{A}_{1}(k)$; here $k[z]_{z}$ is the localization at $z$ of the polynomial algebra $k[z]\left(k[z]_{z}\right.$ is the algebra of Laurent polynomials in $\left.z\right)$, and $\mathbb{A}_{1}(k)$ is the first Weyl algebra. Hence, by [D, 4.5.1 Lemma], the map

$$
\phi_{2}: \operatorname{Spec} \mathcal{U}_{z} \longrightarrow \operatorname{Spec} k[z]_{z}, \quad \phi_{2}(\mathcal{P}):=\mathcal{P} \cap k[z]_{z},
$$

is a homeomorphism. Consequently, the map

$$
\phi:=\phi_{2} \circ \phi_{1}: D(z) \longrightarrow \operatorname{Spec} k[z]_{z}
$$

is a homeomorphism, too. (Note that, by Proposition 3.4 the prime spectrum Spec $k[z]_{z}$ is homeomorphic to $\operatorname{Spec} k[z] \backslash\{\langle z\rangle\}=\{(0)\} \cup\left\{\langle z-\alpha\rangle \mid \alpha \in k^{\times}\right\}$.)

Next, by [D, 2.2.14 Prop.], we know that the quotient algebra $\mathcal{U} / \mathcal{U} z$ is isomorphic to the enveloping algebra of the quotient Lie algebra $\mathfrak{n} / k z$. Also, the map $x+k z \mapsto$ $X, y+k z \mapsto Y$ from $\mathfrak{n} / k z$ to the abelian Lie algebra $k X \oplus k Y$ is obviously a homomorphism of Lie algebras, and so we deduce that $\mathcal{U} / \mathcal{U} z$ is isomorphic to the polynomial algebra $k[X, Y]$ in two (commuting) variables. Finally, since the map

$$
\psi: V(z) \longrightarrow \operatorname{Spec} \mathcal{U} / \mathcal{U} z, \quad \psi(\mathcal{P}):=\mathcal{P} / \mathcal{U} z,
$$

is obviously a homeomorphism, then the following summary of the above is clear. (Note, in particular, that both $D(z)$ and $V(z)$ are homeomorphic to the prime spectra of certain commutative rings; this is a special case of a general phenomenon for the prime spectrum of the enveloping algebra of a solvable Lie algebra BGR, $\S 16]$.)

Lemma B.1. (i) $D(z)$ is homeomorphic to Spec $k[z]_{z}$.

(ii) $V(z)$ is homeomorphic to $\operatorname{Spec} k[X, Y]$.

Having the previous lemma in mind, we derive the next proposition, which gives an explicit description of $\operatorname{Spec} \mathcal{U}$. 
Proposition B.2. For arbitrary $\alpha, \beta, \gamma \in k$, and an irreducible polynomial $F$ from $k[X, Y]$, define the following left ideals of $\mathcal{U}$ :

$$
\begin{aligned}
\mathcal{Q}_{\gamma} & :=\mathcal{U}(z-\gamma), \\
\mathcal{P}^{F(x, y)} & :=\mathcal{U} z+\mathcal{U} F(x, y), \\
\mathcal{M}_{\alpha, \beta} & :=\mathcal{U}(x-\alpha)+\mathcal{U}(y-\beta) .
\end{aligned}
$$

Then:

(i) All the above-defined left ideals are moreover two-sided (pairwise different) prime ideals;

(ii) $\operatorname{Max} \mathcal{U}=\left\{\mathcal{Q}_{\gamma} \mid \gamma \in k^{\times}\right\} \cup\left\{\mathcal{M}_{\alpha, \beta} \mid(\alpha, \beta) \in k^{2}\right\}$;

(iii) $D(z)=\{(0)\} \cup\left\{\mathcal{Q}_{\gamma} \mid \gamma \in k^{\times}\right\}$;

(iv) $V(z)=\left\{\mathcal{Q}_{0}\right\} \cup\left\{\mathcal{P}^{F(x, y)} \mid F \in k[X, Y]\right.$ irreducible $\} \cup\left\{\mathcal{M}_{\alpha, \beta} \mid(\alpha, \beta) \in k^{2}\right\}$;

(v) $\mathcal{M}_{\alpha, \beta}=\mathcal{U} z+\mathcal{U}(x-\alpha)+\mathcal{U}(y-\beta)$.

Proof. To see (v) one just has to note that $(\beta-y)(x-\alpha)+(x-\alpha)(y-\beta)=z$.

Let $P$ and $Q$ be generators of the first Weyl algebra $\mathbb{A}_{1}(k)$ satisfying the relation $[P, Q]=1$. For an arbitrary nonzero $\gamma \in k$, consider the homomorphism of associative algebras $f_{\gamma}: \mathcal{U} \rightarrow \mathbb{A}_{1}(k)$ defined on the generators $x, y, z$ of $\mathcal{U}$ as

$$
f_{\gamma}(x):=\gamma P, \quad f_{\gamma}(y):=Q, \quad f_{\gamma}(z):=\gamma .
$$

Obviously, $f_{\gamma}$ is surjective. Also, it is not hard to see that $\operatorname{ker} f_{\gamma}=\mathcal{Q}_{\gamma}$. Thus, $\mathcal{U} / \mathcal{Q}_{\gamma}$ is isomorphic as an algebra to $\mathbb{A}_{1}(k)$. Now, by [D, 4.6.5 and 4.7.4 Prop.], it is immediate that $\mathcal{Q}_{\gamma} \in \operatorname{Max} \mathcal{U}$.

For the ideals $\mathcal{M}_{\alpha, \beta}$ it can be shown that the quotient algebra $\mathcal{U} / \mathcal{M}_{\alpha, \beta}$ is isomorphic to $k$. Thus, in particular, $\mathcal{M}_{\alpha, \beta}$ are maximal ideals of $\mathcal{U}$.

Combining the above facts and Lemma B.1 we have proved the first four claims of the proposition.

The following theorem is the main result of this appendix.

Theorem B.3. The only $\mathfrak{q}$-stable (equiv. $\mathfrak{l}$-stable) prime ideals of $\mathcal{U}$ are:

$$
(0), \quad \mathcal{Q}_{0}, \quad \mathcal{P}^{x}, \quad \mathcal{P}^{y} \quad \text { and } \quad \mathcal{M}_{0,0}=\mathcal{A} .
$$

In particular, the annihilator primes of an arbitrary module $V$ from the category $\operatorname{Mod}_{\mathfrak{n} \text {-fin }}(\mathfrak{q})$, with $V$ considered as a $\mathcal{U}$-module, are necessarily among the above five primes.

The set of these five distinguished primes of $\mathcal{U}$ can be conveniently presented as the following "q-stable generic sublattice" of $\operatorname{Spec} \mathcal{U}$ (see Figure 1):

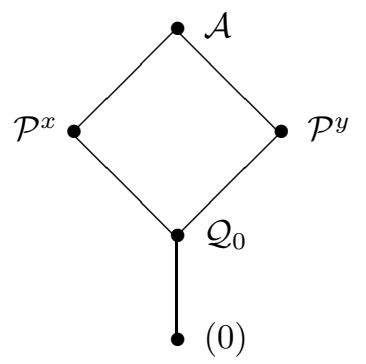

FIGURE 1 
Proof. Define $h(a, b):=a E_{11}+(b-a) E_{22}-b E_{33}$, for any $a, b \in k$; note that $h(a, b) \in \mathfrak{l}$. It is easy to check that

$$
\left[h(a, b), x^{i} y^{j}\right]=((2 a-b) i+(2 b-a) j) x^{i} y^{j},
$$

for all $i, j \in \mathbb{Z}_{+}$.

Obviously, (0) is the only $\mathfrak{q}$-stable prime from $D(z)$. Also, the prime $\mathcal{Q}_{0}$ is $\mathfrak{q}$ stable. Suppose now that $\tilde{\mathfrak{P}} \neq \mathcal{Q}_{0}$ is a $\mathfrak{q}$-stable prime ideal of $\mathcal{U}$ contained in $V(z)$. By Proposition B.2 it is clear that in order to prove the theorem we have to show that either $x$ or $y$ belongs to $\tilde{\mathfrak{P}}$. (Namely, then it follows that $\tilde{\mathfrak{P}} \in\left\{\mathcal{P}^{x}, \mathcal{P}^{y}, \mathcal{M}_{0,0}\right\}$. Further, by Lemma 6.7(ii) we see that all the three listed primes are indeed l-stable; take $\Delta=\emptyset$ and $\Theta=R^{+}$in the lemma.) For the latter, first recall that $\mathcal{U} / \mathcal{Q}_{0}$ may be identified with the polynomial algebra $k[X, Y]$ in two (commuting) variables $X, Y$. Hence, by abuse of notation, we can write $\tilde{\mathfrak{P}}=\mathcal{U} z+\mathcal{U} \mathfrak{P}$ for some $\mathfrak{P} \in \operatorname{Spec} k[x, y]$ (cf. (21)). Let $\vartheta$ be a nontrivial polynomial from $\mathfrak{P}$ of minimal possible degree, say $N$.

Claim. $\vartheta$ is a homogeneous polynomial.

[Decompose $\vartheta=\vartheta_{M}+\cdots+\vartheta_{N}$, where $\vartheta_{j}=\vartheta_{j}(x, y)$ is the homogeneous part of degree $j$, for $0 \leq M \leq j \leq N$; that is, $\vartheta_{j} \in \operatorname{span}_{k}\left\{x^{i} y^{j-i} \mid 0 \leq i \leq j\right\}$. Assume $\vartheta_{M}, \vartheta_{N} \neq 0$ and $M<N$. Now (see (23))

$$
[h(1,1), \vartheta]-N \vartheta=\sum_{i=0}^{N-M-1}(M-N-i) \vartheta_{M+i}
$$

is a nonzero element from $\mathfrak{P}$ of degree less than $N$; a contradiction. Thus $M=N$, and so $\vartheta=\vartheta_{M}$, as we claimed.]

Using the above Claim, we can write $\vartheta=\gamma_{i_{0}} x^{M-i_{0}} y^{i_{0}}+\cdots+\gamma_{j_{0}} x^{M-j_{0}} y^{j_{0}}$, where $i_{0} \leq j_{0}$ and $\gamma_{i_{0}}, \gamma_{j_{0}} \neq 0$. Now, supposing $i_{0}<j_{0}$ and using (23) again, we have that

$$
[h(3,2), \vartheta]-\left(4 M-3 j_{0}\right) \vartheta=\sum_{i=i_{0}}^{j_{0}-1} 3\left(j_{0}-i\right) \gamma_{i} x^{M-i} y^{i}
$$

is a nonzero element from $\mathfrak{P}$. In $j_{0}-i_{0}$ steps we deduce that $x^{M-i_{0}} y^{i_{0}} \in \mathfrak{P}$, and thus by the primeness of $\mathfrak{P}$ we obtain either $x \in \mathfrak{P}$ or $y \in \mathfrak{P}$. This proves the first part of the theorem. The second is then clear because of Theorem C.

The following corollary and its proof are completely analogous to Lemma A.4 and its proof.

Corollary B.4. Let $V$ be a finite-dimensional q-module. Then $\operatorname{Ass} V=\operatorname{Supp}(V)$ $=\{\mathcal{A}\}$, for $V$ considered as a $\mathcal{U}$-module.

We also have the next, very interesting, corollary of the previous theorem (cf. the conjecture in Sect. 6 and Proposition 6.13). First we need one more simple auxiliary lemma.

Lemma B.5. For arbitrary $s, u \in \mathcal{U}$ we have $[s, u] \in \mathcal{Q}_{0}$.

Proof. We may assume that $s$ and $u$ are (normalized) monomials having no powers of $z$; that is, $s=x^{i_{1}} y^{i_{2}}$ and $u=x^{j_{1}} y^{j_{2}}$ for some $i_{1}, i_{2}, j_{1}, j_{2} \in \mathbb{Z}_{+}$. Since $[x, y]=z$, it is clear that the elements $[s, x]$ and $[s, y]$ are from $\mathcal{Q}_{0}$, and so the lemma follows by induction on $j_{1}+j_{2}$. 
Corollary B.6. Let $V$ be a module from the category $\operatorname{Mod}_{\mathfrak{n} \text {-fin }}(\mathfrak{q})$, and $\mathcal{P}$ a nonzero annihilator prime of $V$ when $V$ is considered as a $\mathcal{U}$-module; that is, $\mathcal{P}$ is from the set $\left\{\mathcal{Q}_{0}, \mathcal{P}^{x}, \mathcal{P}^{y}, \mathcal{A}\right\}$. Suppose that $v \in V$ is a nonzero vector satisfying $\mathcal{P}=\mathfrak{A}_{v}$. Then

(i) $\mathcal{P}=\operatorname{ann}_{\mathcal{U}}(v)$;

(ii) $\mathcal{P} \in \operatorname{Supp}(V)$.

As a consequence, we also have $\mathcal{A} V \neq V$.

Proof. (i) Suppose that $s v=0$ for some $s \in \mathcal{U} \backslash \mathcal{P}$. Using the fact that $\mathcal{Q}_{0} \subseteq \mathcal{P}$ and Lemma B.5. we immediately obtain $s u v=0$, for every $u \in \mathcal{U}$. Thus, it follows that $s \in \operatorname{ann}_{\mathcal{U}}(\mathcal{U} v)=\mathcal{P}$; a contradiction.

Obviously, (ii) is a direct consequence of (i). Also, the last claim follows by (ii), Lemma 2.8 and Theorem 2.10 i).

Remark B.7. (1) The assumption $\mathcal{P} \neq(0)$ in the above corollary cannot be dropped. To see this, take $V$ to be the $k$-vector space $\bigoplus k v_{i, j}$, the (direct) sum over $i \in \mathbb{Z}_{+}$, $j \in \mathbb{Z}$. Stafford and Wallach [SW] Sect. 4] defined a series of $\mathfrak{g}$-module structures on $V$ parametrized by $\alpha \in k$. In every $\mathfrak{g}$-module from this series $\mathfrak{n}$ has the same action, which is given as follows:

$$
x v_{i, j}:=v_{i+1, j}, \quad y v_{i, j}:=v_{i, j+1}-i v_{i-1, j-1}, \quad z v_{i, j}:=v_{i, j-1} .
$$

Now, combining the facts $\mathcal{U} v_{0,0}=V$ and $z V=V$ noted in [SW], Thm. 4.2] and the previous corollary, we deduce that $(0)$ is the only annihilator prime of $V$ as a $\mathcal{U}$-module, and at the same time $(0) \notin \operatorname{Supp}(V)$. Moreover, we have the following somewhat surprising fact. (This once more emphasizes the significant difference between the commutative and noncommutative theories.)

Fact. $\operatorname{Supp}(V)=\emptyset$, for $V$ considered as a $\mathcal{U}$-module.

[Clearly, we have to show that $\mathcal{Q}_{\gamma} \notin \operatorname{Supp}(V)$, for every $\gamma \in k^{\times}\left(\mathcal{Q}_{\gamma}\right.$ as in Proposition B.2). For that purpose, take an arbitrary $v \in V$ and suppose $n_{0} \in \mathbb{N}$ is such that in the decomposition $v=\sum c_{i j} v_{i, j}, c_{i j} \in k$, we have $c_{i j}=0$ for $i \geq n_{0}$. By denoting $\sigma:=y z-1, s:=\sigma^{n_{0}}$ and using the equality $\sigma v_{i, j}=-i v_{i-1, j-1}$ (use (24)), we deduce that $s v=0$. It remained to observe that $s \notin \mathcal{Q}_{\gamma}$; the latter follows by [D, 4.6.3] and the fact that $f_{\gamma}(\sigma)=\gamma Q-1$ (see (22)).]

(2) Note that the module $V$ in (1), considered as a $\mathcal{U}$-module, is faithful. [Indeed, otherwise we would have that every foundation, and thus also every annihilator, prime of $V$ is a nonzero ideal (see [Š2, Lemma 3.8(i) and Cor. 3.7(i)]). But this contradicts the previously obtained conclusion Annspec $V=\{(0)\}$.] Related to this, note the following. If $V$ is a finitely generated unfaithful module over the enveloping algebra of an arbitrary nilpotent Lie algebra, then $(0) \notin \operatorname{Supp}(V)$; that is, the localization of $V$ at (0) is trivial (cf. Lemma 3.9).

We will conclude this appendix with the analog of Fact A.6 For that purpose, define the algebraic groups

$$
L:=\left\{\left(\begin{array}{ccc}
\tau_{1} & 0 & 0 \\
0 & \tau_{2} & 0 \\
0 & 0 & \tau_{1}^{-1} \tau_{2}^{-1}
\end{array}\right) \mid \tau_{1}, \tau_{2} \in k^{\times}\right\}
$$


and

$$
N:=\left\{\left(\begin{array}{ccc}
1 & a & c \\
0 & 1 & b \\
0 & 0 & 1
\end{array}\right) \mid a, b, c \in k\right\} .
$$

Then the group $Q:=L N$ acts on $\mathfrak{q}$ via the adjoint action (see (12)). We want to consider the coadjoint action of $Q$ on the dual $\mathfrak{n}^{*}$ of $\mathfrak{n}$, and then describe the corresponding orbits $Q . \varphi, \varphi \in \mathfrak{n}^{*}$ (see (15)). Note that in order to compute these orbits we may first compute the $N$-orbits $N . \varphi, \varphi \in \mathfrak{n}^{*}$, that is, the orbit space $\mathfrak{n}^{*} / N$, and then compute the $L$-orbits $L . \psi, \psi \in \mathfrak{n}^{*} / N$. Now, let $\left(x^{*}, y^{*}, z^{*}\right)$ be the dual basis of the basis $(x, y, z)$ of $\mathfrak{n}$; then $\mathfrak{n}^{*}=k x^{*} \oplus k y^{*} \oplus k z^{*}$. In the sequel we will identify $\mathfrak{n}^{*}$ with $k^{3}$ via this dual basis. Then we have the following well known description of $\mathfrak{n}^{*} / N\left(\equiv k^{3} / N\right)$ :

$$
\mathfrak{n}^{*} / N=\left\{\left(u_{0}, v_{0}, 0\right) \mid u_{0}, v_{0} \in k\right\} \cup\left\{\mathcal{H}_{w_{0}} \mid w_{0} \in k^{\times}\right\} ;
$$

here $\mathcal{H}_{w_{0}}$ denotes the linear hyperplane $\left(k, k, w_{0}\right)$. Having this, it is easy to check that $L .\left(u_{0}, 0,0\right)=\left(k^{\times}, 0,0\right), L .\left(0, v_{0}, 0\right)=\left(0, k^{\times}, 0\right)$ and $L .\left(u_{0}, v_{0}, 0\right)=\left(k^{\times}, k^{\times}, 0\right)$, for $u_{0}, v_{0} \in k^{\times}$. Also, we have $L . \mathcal{H}_{w_{0}}=\left(k, k, k^{\times}\right)$, for $w \in k^{\times}$. Summarizing, we obtained the following description of all the $Q$-orbits on $\mathfrak{n}^{*}$.

Fact B.8. The only (coadjoint) Q-orbits on $\mathfrak{n}^{*}$ (that is, the points of the orbit space $\left.\mathfrak{n}^{*} / Q\right)$ are:

$$
(0,0,0), \quad\left(k^{\times}, 0,0\right), \quad\left(0, k^{\times}, 0\right), \quad\left(k^{\times}, k^{\times}, 0\right), \quad\left(k, k, k^{\times}\right) .
$$

The set of closures of the above five orbits, in the Zariski topology on $\mathfrak{n}^{*}$, can be nicely presented by the following figure. (Note that if $\mathcal{O}_{1}, \mathcal{O}_{2}$ are any two of the listed orbits, then for their closures $\overline{\mathcal{O}}_{1}, \overline{\mathcal{O}}_{2}$ we have $\overline{\mathcal{O}}_{1} \supseteq \overline{\mathcal{O}}_{2}$ if and only if $\overline{\mathcal{O}}_{1}$ is above $\overline{\mathcal{O}}_{2}$ in Figure 2.)

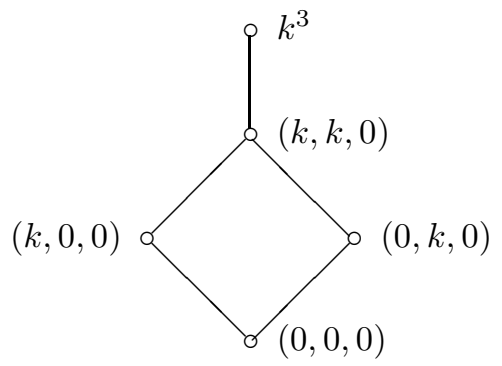

FiguRE 2

Added note. The author is grateful to all the referees for careful reading of various versions of the manuscript. Their numerous remarks and suggestions significantly improved the author's original version(s) in style and/or clarity of exposition.

\section{REFERENCES}

[BB] A. Beilinson and J. Bernstein, A generalization of Casselman's submodule theorem, Representation Theory of Reductive Groups (Peter C. Trombi, ed.), Progress in Mathematics, vol. 40, Birkhäuser, Boston, 1983, pp. 35-52. MR 85e:22024

[Bh] W. Borho, A survey on enveloping algebras of semisimple Lie algebras, Lie Algebras and Related Topics, CMS Conf. Proc., vol. 5, Amer. Math. Soc., Providence, RI, 1986, pp. 19-50. MR 87g:17013 
[BGR] W. Borho, P. Gabriel and R. Rentschler, Primideale in Einhüllenden auflösbarer LieAlgebren, Lecture Notes in Math. 357, Springer-Verlag, Berlin, 1973. MR 51:12965

[B1] N. Bourbaki, Commutative Algebra, Hermann, Paris, and Addison Wesley, Reading, Massachusetts, 1972. MR 50:12997

[B2] _ Algèbre, Chapitres I,II,III, Chapitres VII, VIII, Hermann, Paris, 1970. MR 43:2

[B3] Groupes et Algèbres de Lie, Chapitres IV,V,VI, Hermann, Paris, 1968. MR 39:1590

[C] W. Casselman, Jacquet modules for real reductive groups, Proc. Int. Cong. Math., Helsinki (1978), pp. 557-563. MR 83h:22025

[CM] W. Casselman and D. Miličić, Asymptotic behavior of matrix coefficients of admissible representations, Duke Math. J. 49 (1982), 869-930. MR 85a:22024

[CO] W. Casselman and M. S. Osborne, The restriction of admissible representations to $\mathfrak{n}$, Math. Ann. 233 (1978), 193-198 MR 58:1033.

[D] J. Dixmier, Enveloping Algebras, North-Holland Mathematical Library, Vol. 14, NorthHolland, Amsterdam, 1977. MR 58:16803b

[GW] K. R. Goodearl and R. B. Warfield, Jr., An Introduction to Noncommutative Noetherian Rings, "London Math. Soc. Stud. Texts", Vol. 16, Cambridge Univ. Press, Cambridge, 1989. MR 91c:16001

[HT] R. Howe and E. C. Tan, Non-Abelian Harmonic Analysis, Applications of $\mathrm{SL}(2, \mathbb{R})$, Springer-Verlag, New York, 1992. MR 93f:22009

[J] A. V. Jategaonkar, Localization in Noetherian Rings, "London Math. Soc. Lect. Note Ser.", Vol. 98, Cambridge Univ. Press, Cambridge, 1986. MR 88c:16005

[K] A. W. Knapp, Lie Groups Beyond an Introduction, Progress in Mathematics, vol. 140, Birkhäuser, Boston, 1996. MR 98b:22002

[McC] J. C. McConnell, The intersection theorem for a class of non-commutative rings, Proc. London Math. Soc. (3) 17 (1967), 487-498. MR 35:1624

[MR] J. C. McConnell and J. C. Robson, Noncommutative Noetherian rings, J. Wiley, New York, 1987. MR 89j:16023

[NG] Y. Nouazé and P. Gabriel, Idéaux premiers de l'algèbre enveloppante d'une algèbre de Lie nilpotente, J. Algebra 6 (1967), 77-99. MR 34:5889

[S] P. F. Smith, Localization and the AR property, Proc. London Math. Soc. (3) 22 (1971), 39-68. MR 45:3453

[St] J. T. Stafford, On the regular elements of Noetherian rings, Ring Theory, Proceedings of the 1978 Antwerp Conference (F. Van Oystaeyen, ed.), Lecture Notes in Pure and Appl. Math., vol. 51, Marcel Dekker, New York, 1979, pp. 257-277. MR 81k:16016

[SW] J. T. Stafford and N. R. Wallach, The restriction of admissible modules to parabolic subalgebras, Trans. Amer. Math. Soc. 272 (1982), 333-350. MR 83h:17007

[ک̌1] B. Širola, On associated primes and weakly associated primes, Interactions Between Ring Theory and Representations of Algebras, Proceedings of the 1998 Murcia Conference (F. Van Oystaeyen and M. Saorin, eds.), M. Dekker, New York (2000), pp. 383-391. CMP 2000:13

[క̌2] - Annihilator primes and foundation primes, preprint.

[ک̌3] _ Going up for the enveloping algebras of Lie algebras, submitted.

[Š4] On noncommutative Noetherian schemes, preprint.

[V1] D. A. Vogan, Jr., The orbit method and primitive ideals for semisimple Lie algebras, Lie Algebras and Related Topics, CMS Conf. Proc., vol. 5, Amer. Math. Soc., Providence, RI, 1986, pp. 281-316. MR 87k:17015

[V2] Associated varieties and unipotent representations, Harmonic Analysis on Reductive Groups (W. Barker and P. Sally, eds.), Birkhäuser, Boston, 1991, pp. 315-388. MR 93k:22012

Department of Mathematics, Univerity of Zagreb, PP 335, 10002 Zagreb, Croatia

E-mail address: sirola@math.hr 